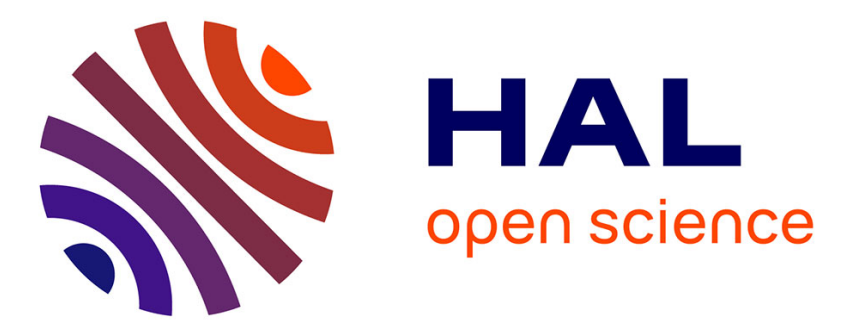

\title{
Extratropical Low-Frequency Variability With ENSO Forcing: A Reduced-Order Coupled Model Study
}

Stéphane Vannitsem, Jonathan Demaeyer, Michael Ghil

\section{To cite this version:}

Stéphane Vannitsem, Jonathan Demaeyer, Michael Ghil. Extratropical Low-Frequency Variability With ENSO Forcing: A Reduced-Order Coupled Model Study. Journal of Advances in Modeling Earth Systems, 2021, 13 (6), 10.1029/2021ms002530 . hal-03288068

\section{HAL Id: hal-03288068 https://hal.sorbonne-universite.fr/hal-03288068}

Submitted on 16 Jul 2021

HAL is a multi-disciplinary open access archive for the deposit and dissemination of scientific research documents, whether they are published or not. The documents may come from teaching and research institutions in France or abroad, or from public or private research centers.
L'archive ouverte pluridisciplinaire HAL, est destinée au dépôt et à la diffusion de documents scientifiques de niveau recherche, publiés ou non, émanant des établissements d'enseignement et de recherche français ou étrangers, des laboratoires publics ou privés. 


\section{AMES Journal of Advances in Modeling Earth Systems}

\author{
RESEARCH ARTICLE \\ 10.1029/2021MS002530 \\ Key Points: \\ - Two pullback attractors (PBAs) \\ coexist in the model's midlatitudes \\ for both periodic and chaotic $\mathrm{El}$ \\ Niño-Southern Oscillation (ENSO) \\ forcing \\ - These local PBAs are nonlinearly \\ unstable, with some trajectories that \\ visit both of them \\ - The ENSO forcing synchronizes the \\ midlatitude behavior in unexpected \\ ways
}

Correspondence to:

S. Vannitsem,

Stephane.Vannitsem@meteo.be

Citation:

Vannitsem, S., Demaeyer, J., \& Ghil, M. (2021). Extratropical low-frequency variability with ENSO forcing: A reduced-order coupled model study. Journal of Advances in Modeling Earth Systems, 13, e2021MS002530. https:// doi.org/10.1029/2021MS002530

Received 2 MAR 2021 Accepted 14 MAY 2021 (c) 2021. The Authors. Journal of Advances in Modeling Earth Systems published by Wiley Periodicals LLC on behalf of American Geophysical Union. This is an open access article under the terms of the Creative Commons Attribution-NonCommercial License, which permits use, distribution and reproduction in any medium, provided the original work is properly cited and is not used for commercial purposes.

\section{Extratropical Low-Frequency Variability With ENSO Forcing: A Reduced-Order Coupled Model Study}

\author{
Stéphane Vannitsem $^{1}$ (D), Jonathan Demaeyer ${ }^{1}$ iD, and Michael Ghil ${ }^{2,3}$ \\ ${ }^{1}$ Royal Meteorological Institute of Belgium, Brussels, Belgium, ${ }^{2}$ Geosciences Department and Laboratoire de \\ Météorologie Dynamique (CNRS and IPSL), Ecole Normale Supérieure and PSL University, Paris, France, ${ }^{3}$ Department \\ of Atmospheric \& Oceanic Sciences, University of California at Los Angeles, Los Angeles, CA, USA
}

Abstract The impact of the El Niño-Southern Oscillation (ENSO) on the extratropics is investigated in an idealized, reduced-order model that has a tropical and an extratropical module. Unidirectional ENSO forcing is used to mimick the atmospheric bridge between the tropics and the extratropics. The variability of the coupled ocean-atmosphere extratropical module is then investigated through the analysis of its pullback attractors (PBAs). This analysis focuses on two types of ENSO forcing generated by the tropical module, one periodic and the other aperiodic. For a substantial range of the ENSO forcing, two chaotic PBAs are found to coexist for the same set of parameter values. Different types of extratropical low-frequency variability (LFV) are associated with either PBA over the parameter ranges explored. For periodic ENSO forcing, the coexisting PBAs exhibit only weak nonlinear instability. For chaotic forcing, though, they are quite unstable and certain extratropical perturbations induce transitions between the two PBAs. These distinct stability properties may have profound consequences for extratropical climate predictions: in particular, ensemble averaging may no longer help isolate the LFV signal.

Plain Language Summary The authors have investigated the variability of a simplified coupled ocean-atmosphere model for the Earth's midlatitudes, subject to the influence of the El NiñoSouthern Oscillation (ENSO). This study reveals that multiple climates may coexist, each of which is characterized by distinct types of low-frequency variability (LFV) and predictability properties. When the ENSO forcing is periodic, these climates are fairly robust against perturbations, but when it is chaotic, small perturbations induce transitions between the different climates. These properties could have profound consequences for extratropical climate predictions, since ensemble averaging may no longer be a valid approach to ascertain the LFV signal.

\section{Introduction and Motivation}

The Earth system is a dissipative multiscale system forced by external time-dependent signals of various origins (Ghil \& Lucarini, 2020). Given the system's enormous complexity, one often splits it into subsystems, each of which is affected by the others. Each subsystem can, in turn, be considered as being both forced and dissipative (Ghil \& Childress, 1987; Lorenz, 1963). The description of their evolution relies on the use of conservation laws, combined with appropriate simplifications, subject to the influence of the external forcings. The dynamics of such systems can be described using concepts and methods from dynamical systems theory.

Recent developments in this theory have addressed the effect of time-dependent external forcing, and are now organized in the theory of nonautonomous (NDSs: Carvalho et al., 2012; Kloeden \& Rasmussen, 2011) and random (RDSs: Arnold, 1998; Caraballo \& Han, 2017) dynamical systems. This theory has been applied to the atmospheric, oceanic, and climate sciences over the last dozen years or so by several groups of authors (Ashwin et al., 2012; Checkroun et al., 2011, 2018, 2018; Ditlevsen \& Ashwin, 2018; Drótos et al., 2015, 2016; Ghil et al., 2008; Pierini, 2020; Pierini et al., 2016, 2018; Sévellec \& Fedorov, 2015; Tél et al., 2020).

An important characteristic of systems with time-dependent external forcing or coefficients is that one can no longer assume ergodicity, and thus temporal averages are no longer good approximations to ensemble averages. When periodic forcing is acting, the usual ergodicity property has to be generalized to cycloergodicity, which requires temporal averages to be performed separately at each moment of the cycle. 
When the forcing is erratic-that is, either random or chaotic-there is no equivalence between the two types of averages (Caraballo \& Han, 2017; Checkroun et al., 2011; Drótos et al., 2016). Moreover, as will be seen in the current work, multiple types of qualitatively different solutions may be present for the same parameter values, complicating further the description of the system (Pierini et al., 2016, 2018; Pierini \& Ghil, 2021). The tools to explore the dynamics of the atmosphere, ocean, and coupled climate system in these cases are provided by NDS and RDS theory and are known, alternatively, as pullback attractors (PBAs: Checkroun et al., 2011; Ghil et al., 2008) or snapshot attractors (Drótos et al., 2015, 2016).

An important result in this setting is that, in the presence of either periodic or irregular forcing, multiple PBAs can coexist within a unique global attractor. In particular, Pierini et al. (2016), Pierini et al. (2018), and Pierini and Ghil (2021) have considered a severely truncated model of wind-driven, midlatitude ocean dynamics in which two PBAs coexist: one of the two exhibit rather quiescent, small-amplitude behavior, while the behavior of the other one is much more energetic and irregular, for exactly the same prescribed time-dependent forcing. As we shall see in the present work, such a multiplicity of solution regimes is not limited to highly idealized, low-order models.

For several decades, the influence of the El Niño-Southern Oscillation (ENSO) on the midlatitude and high latitude of both hemispheres has been an important area of climate studies (McPhaden et al., 2020; Philander, 1990). This area's importance is largely due to the presence of teleconnections between the Tropical Pacific and many regions all over the world (Alexander et al., 2002; Hoerling \& Kumar, 2002; López-Parages et al., 2016; Schemm et al., 2018). A particularly important question is exploring the impact of ENSO forcing on the predictability of extratropical climate (Kumar \& Hoerling, 1995; Nidheesh et al., 2017).

Most of the above-mentioned studies have relied on comprehensive, high-end models for their analyses. A major difficulty in this case is the limited number of model runs that can be performed, due to the large computational cost of each run (Ghil, 2001; Held, 2005). Moreover, these runs usually start from initial states that are not very far in the past and so the solutions do not necessarily sample the correct asymptotic behavior. This state of affairs also implies that one gets, at best, only a very partial view of a given model's solution space. These obstacles can be overcome by using first simpler models that will provide hints on the possible solutions generated by the use of a vastly larger number of parameter values and initial states, thus providing crucial guidance for future simulations and predictions with larger models.

This study aims to explore the possible existence of multiple types of extratropical climate trajectories that are compatible with a given type of ENSO forcing. This exploration is performed in the setting of a reduced-order, coupled tropical-extratropical model, and it relies on large numbers of runs and on sophisticated methods for analyzing them. The ENSO forcing studied is either periodic or chaotic, and it covers a wide range of intensities: several PBAs are found for a given type and intensity of the forcing. The stability properties of these PBAs are explored, showing that some are unstable and that model trajectories may transit from one PBA's attractor basin to another.

Section 2 describes the coupled tropical-extratropical model. In Section 3, we construct the PBAs and study their properties. Section 4 summarizes the main results and the key messages to keep in mind for ensemble forecasting and climate projections.

\section{Governing Equations for the Tropical-Extratropical Model}

\subsection{The ENSO Module}

The ENSO model used herein was developed in a series of papers by Jin (1996, 1997), An and Jin (2004), Timmermann et al. (2003), and Roberts et al. (2016). They modeled the dynamics of the ocean's upper layer in the Tropical Pacific using a low number of variables. Their two-box ENSO model describes the dynamics of the temperature in the eastern and western Tropical Pacific basins, and it is completed by an equation for the evolution of the thermocline depth. The model represents the horizontal discharge-recharge mechanisms at play in the Tropical Pacific through the heat exchanges between the tropical and subtropical waters, subject to surface wind stress and upwelling of subsurface cold water in the eastern part of the domain (Jin, 1997). 
Table 1

Dimensionless Parameter Values of the ENSO Module

\begin{tabular}{ll}
\hline Periodic & \multicolumn{1}{c}{ Chaotic } \\
\hline$a=6.8927$ & $a=7.658609809$ \\
$\rho=0.3224$ & $\rho=0.29016$ \\
$\delta=0.00028058$ & $\delta=0.0002803$ \\
$c=2.3952$ & $c=2.3952$ \\
$k=0.4032$ & $k=0.4032$ \\
$s=0.0010691$ & $s=0.001069075$ \\
\hline
\end{tabular}

Abbreviation: ENSO, El Niño-Southern Oscillation.
Roberts et al. (2016) introduced a nondimensional model version in which the time, for instance, is normalized by a typical time scale of tropical wave propagation of roughly 3.5 months or 105 days. The latter ENSO model version is coupled here with an extratropical module whose time is nondimentionalized by the Coriolis parameter $f_{0}$, and the Roberts et al. (2016) equations are slightly modified therefore.

The nondimensional equations governing our ENSO module are, accordingly:

$$
\begin{aligned}
\frac{d X}{d t} & =\rho \delta\left(X^{2}-a X\right)+s X(X+Y+c-c \tanh (X+Z)) \\
\frac{d Y}{d t} & =-\rho \delta\left(a Y+X^{2}\right) \\
\frac{d Z}{d t} & =\delta\left(k-Z-\frac{X}{2}\right)
\end{aligned}
$$

Here, $X, Y, Z$, and $t$ are dimensionless, $X$ is the temperature difference between the eastern and western basins of the Tropical Pacific, $Y$ the western basin's temperature anomaly with respect to a reference value, and $Z$ the western basin's thermocline depth anomaly.

The dimensionless parameters are defined as follows

$$
\begin{array}{lll}
a=\frac{\alpha b L}{\epsilon h \beta}, & \rho=\frac{\epsilon h \beta}{r b L}, & \delta=\frac{r}{f_{0}}, \\
c=\frac{C}{S_{0}}, & k=\frac{K}{S_{0}}, & s=\frac{\zeta h \beta}{b L f_{0}},
\end{array}
$$

with $f_{0}$ the Coriolis parameter used to nondimensionalize the time, and the other parameters as defined in Roberts et al. (2016, Table 1). These parameters are expressed as mathematically convenient combinations of physical parameters. The two parameters $1 / \delta$ and $\rho$ are proportional to the dynamical adjustment time scale of the thermocline depth, while $1 / a$ is proportional to the thermal damping time scale of the eastern and western equatorial temperatures. The parameter $c$ is proportional to the maximum temperature range in the model, while $k$ is proportional to a reference depth. The parameter $s$ appears in the current model due to our change of normalization of time, and it is proportional to the vertical advection strength $\zeta$.

For the present study, we concentrated on two sets of parameter values. These two sets lead to either a periodic or a chaotic solution, and they are listed in Table 1 below. The first set is taken from Timmermann et al. (2003) and it leads to amplitude-modulated oscillatory solutions that arise beyond a period doubling bifurcation as the efficiency $\epsilon$ of zonal advection is increased. The chaotic solution is taken from the analysis of Guckenheimer et al. (2017) on the development of mixed-mode oscillations. For certain parameter values, multiple attractors exist and have complicated basins of attractions. The initial condition used to find the chaotic solution used here is $\left(X_{0}, Y_{0}, Z_{0}\right)=(-2.8439,-0.62,1.48)$.

The ENSO module is taken here to be unaffected by the midlatitude module, and is thus the driving (or master) system in our coupled model, in the sense of the skew products of Sell (1971); see also Kloeden and Rasmussen (2011), Caraballo and Han (2017) and Ghil and Lucarini (2020, Section III.G). Equation 1 can thus be integrated independently of the rest of the model, and this is done using a fourth-order Runge-Kutta scheme with a time step $\Delta t=0.1346 h=0.05$ nondimensional time units. See the previous page for nondimensionalization, just below Equation 2.

The results of such integrations are shown in Figures $1 \mathrm{a}$ and $1 \mathrm{~b}$ for the periodic and the chaotic case, respectively. The variable $X+Y$ plotted in the figure corresponds in our simple model (1) to the sea surface temperatures in the eastern Tropical Pacific that are commonly associated with the Niño-3 index. 

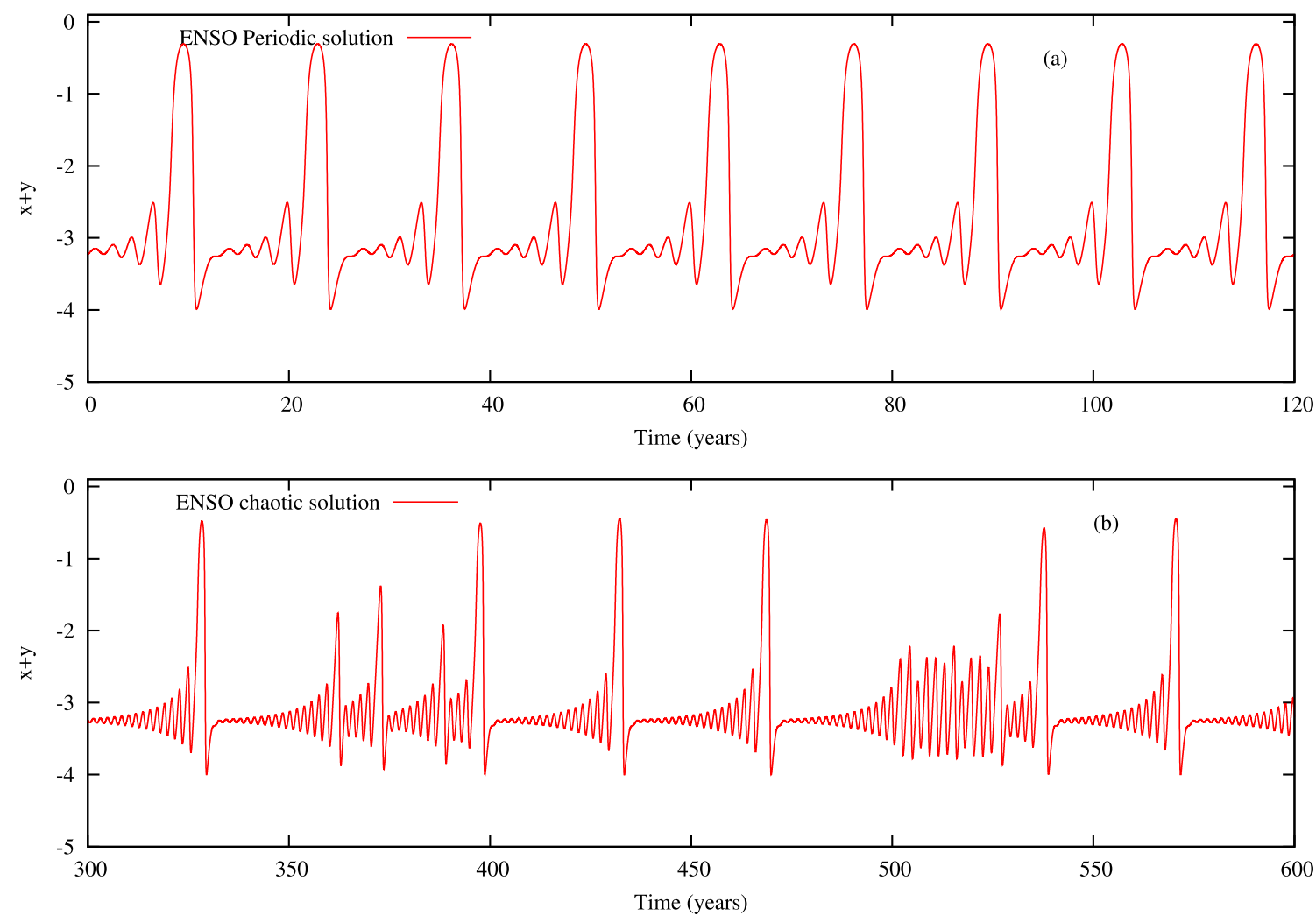

Figure 1. Trajectory segments of the ENSO model for the eastern Tropical Pacific basin's temperature anomalies $X+Y$. (a) Periodic case, and (b) chaotic case; see Equation 1 and Table 1 for details. The bursting behavior in both cases, with very large excursions toward more positive values, occurs periodically in panel (a) and irregularly in panel (b). Notice that the total length of the segments is 120 years in panel (a) and 300 years in panel (b). ENSO, El Niño-Southern Oscillation.

The periodic solution for the ENSO forcing in Figure 1a agrees qualitatively with the "bursting" behavior emphasized by Timmermann et al. (2003). Thus, large warm events in the Niño-3 area alternate with several cycles of moderate warm-and-cold events, with a periodicity of roughly 10 years (Timmermann et al., 2003, Figure 11b).

A long chaotic solution was started from the initial state $(X=-2.8439, Y=-0.62, Z=1.480)$, with an integration length of $20,000,000$ time units that corresponds to about 6,145 years; that is, 10,000,000 time units $\simeq 3,072$ years. In general, such runs were equally divided into a transient portion to reach the asymptotic behavior and an analysis portion; in the present case, both portions were equal to 10,000,000 time units.

A 300-year segment of the chaotic solution's analysis interval is plotted in Figure 1b. The large bursts occur much less frequently here, on less realistic time scales of the order of 30-50 years. On the other hand, the evolution in this case is more erratic - with much greater irregularity in the timing of the bursts-as seen in observations and in other chaotic ENSO models (e.g., Jin et al., 1994, 1996; Tziperman et al., 1994).

\subsection{The VDDG Extratropical Model}

The coupled ocean-atmosphere model used herein for the midlatitudes was developed by Vannitsem et al. (2015) and it is called hereafter the VDDG model. Different versions of this VDDG model have been used in the study of LFV within the coupled ocean-atmosphere system (De Cruz et al., 2016; Vannitsem, 2017), the properties of the Lyapunov exponents in such a system (De Cruz et al., 2018; Vannitsem \& Lucarini, 2016), the stochastic parametrization of subgrid-scale forcing (Demaeyer \& Vannitsem, 2018), and data assimilation in coupled models (Penny et al., 2019; Tondeur et al., 2020; Carrassi et al., 2021). 
Our coupled model's atmospheric module is based on the vorticity equations of a two-layer quasi-geostrophic flow defined on a beta-plane (Gill, 1982; Pedlosky, 1987). The equations in pressure coordinates are:

$$
\begin{gathered}
\frac{\partial}{\partial t}\left(\nabla^{2} \psi_{a}^{1}\right)+J\left(\psi_{a}^{1}, \nabla^{2} \psi_{a}^{1}\right)+\beta \frac{\partial \psi_{a}^{1}}{\partial x}=-k_{d}^{\prime} \nabla^{2}\left(\psi^{1}-\psi^{3}\right)+\frac{f_{0}}{\Delta p} \omega, \\
\frac{\partial}{\partial t}\left(\nabla^{2} \psi_{a}^{3}\right)+J\left(\psi_{a}^{3}, \nabla^{2} \psi_{a}^{3}\right)+\beta \frac{\partial \psi_{a}^{3}}{\partial x}=+k_{d}^{\prime} \nabla^{2}\left(\psi_{a}^{1}-\psi_{a}^{3}\right)-\frac{f_{0}}{\Delta p} \omega-k_{d} \nabla^{2}\left(\psi_{a}^{3}-\psi_{o}\right) .
\end{gathered}
$$

Here, $\psi_{a}^{1}$ and $\psi_{a}^{3}$ are the streamfunction fields at 250 and $750 \mathrm{hPa}$, respectively, while $\omega=d p / d t$ is the vertical velocity, $f_{0}$ the Coriolis parameter at $\phi_{0}=45$ latitude, and $\beta=d f / d y$ at $\phi_{0}$. The coefficients $k_{d}$ and $k_{d}^{\prime}$ multiply the surface friction term and the internal friction between the layers, respectively.

An additional term has been introduced in Equation $3 \mathrm{~b}$ to account for the presence of a surface boundary velocity $\psi_{o}$ of the oceanic flow; see Equation 4 below. This term corresponds to the Ekman pumping on a moving surface and is the mechanical contribution of the interaction between the ocean and the atmosphere.

The coupled model's ocean dynamics is described by the reduced-gravity, quasi-geostrophic shallow-water model (Gill, 1982; Pedlosky, 1987). The forcing is provided by the wind generated by the atmospheric module above. The governing equation is:

$$
\frac{\partial}{\partial t}\left(\nabla^{2} \psi_{o}-\frac{\psi_{o}}{L_{R}^{2}}\right)+J\left(\psi_{o}, \nabla^{2} \psi_{o}\right)+\beta \frac{\partial \psi_{o}}{\partial x}=-r \nabla^{2} \psi_{o}+\frac{\operatorname{curl}_{z} \vec{\tau}}{\rho_{o} H},
$$

where $\psi_{o}$ is the streamfunction, $\rho_{o}$ the density of water, $H$ the depth of the fluid layer, $L_{R}$ the reduced Rossby deformation radius, $r$ a Rayleigh friction coefficient at the bottom of the fluid layer, and $\operatorname{curl}_{z} \vec{\tau}$ is the vertical component of the wind stress curl.

The wind stress in the VDDG model is given by $\left(\tau_{x}, \tau_{y}\right)=C(u-U, v-V)$, where $\left(u=-\partial \psi_{\mathrm{a}}^{3} / \partial y, v=\partial \psi_{\mathrm{a}}^{3} / \partial x\right)$ are the horizontal components of the geostrophic wind, and $(U, V)$ the components of the geostrophic currents in the ocean. One thus gets

$$
\operatorname{curl}_{z} \tau=C \nabla^{2}\left(\psi_{\mathrm{a}}^{3}-\psi_{\mathrm{o}}\right) .
$$

and the wind stress is proportional to the relative velocity between the flow in the ocean's upper layer and the wind in the lower atmospheric layer. The drag coefficient $d=C /\left(\rho_{0} H\right)$ gives the strength of the mechanical coupling between the ocean and the atmosphere and it was a key bifurcation parameter in Vannitsem et al. (2015). Here, $C$ will also play a crucial role in affecting the VDDG model's behavior subject to ENSO forcing.

The dynamic Equations 3 and 4 are supplemented by temperature equations for the two subsystems. For the atmosphere,

$$
\gamma_{a}\left(\frac{\partial T_{a}}{\partial t}+J\left(\psi_{a}, T_{a}\right)-\sigma \omega \frac{p}{R}\right)=-\lambda\left(T_{a}-T_{o}\right)+E_{a, R}
$$

with

$$
E_{a, R}=\epsilon_{a} \sigma_{B} T_{o}^{4}-2 \epsilon_{a} \sigma_{B} T_{a}^{4}+R_{a}
$$

Here, $T_{a}$ and $T_{o}$ are the atmospheric and oceanic temperatures, respectively, $R$ is the gas constant, $\epsilon_{a}$ the emissivity of the atmosphere, $\sigma_{B}$ the Stefan-Boltzman constant, $R_{a}$ the shortwave radiation entering the atmosphere, $\omega$ the vertical velocity in pressure coordinates, $\gamma_{a}$, the heat capacity of the atmosphere, $p$ the pressure, and $\lambda$ is the inverse of the time scale associated with heat transfer between the ocean and the atmosphere, which includes both the latent and sensible heat fluxes. In fact we assume that this combined heat transfer is proportional to the temperature difference between the atmosphere and the ocean. The 
Table 2

List of Parameters of the Extratropical VDDG Module

\begin{tabular}{lccc}
\hline Parameter (unit) & Value & Parameter (unit) & Value \\
\hline$L_{y}=\pi \mathrm{L}(\mathrm{km})$ & $5.0 \times 10^{3}$ & $\gamma_{o}\left(\mathrm{~J} \mathrm{~m}^{-2} \mathrm{~K}^{-1}\right)$ & $4 \times 10^{6} \mathrm{H}$ \\
$f_{0}\left(\mathrm{~s}^{-1}\right)$ & $1.032 \times 10^{-4}$ & $C_{o}\left(\mathrm{~W} \mathrm{~m}^{-2}\right)$ & 310 \\
$n=2 \mathrm{~L}_{\mathrm{y}} / \mathrm{L}_{\mathrm{x}}$ & 1.5 & $T_{\mathrm{o}}^{0}(\mathrm{~K})$ & 285 \\
$R_{E}(\mathrm{~km})$ & 6,370 & $\gamma_{a}\left(\mathrm{~J} \mathrm{~m}^{-2} \mathrm{~K}^{-1}\right)$ & $1.0 \times 10^{7}$ \\
$\phi_{0}$ & $\pi / 4$ & $\left.C_{a}(\mathrm{~W} \mathrm{~m})^{-2}\right)$ & $C_{\mathrm{o}} / 4$ \\
$g^{\prime}$ & $3.10 \times 10^{-2}$ & $\epsilon_{a}$ & 0.76 \\
$r\left(\mathrm{~s}^{-1}\right)$ & $1.0 \times 10^{-7}$ & $\beta\left(\mathrm{m}^{-1} \mathrm{~s}^{-1}\right)$ & $1.62 \times 10^{-11}$ \\
$H(\mathrm{~m})$ & 100 & $T_{\mathrm{a}}^{0}(\mathrm{~K})$ & 270 \\
$d\left(\mathrm{~s}^{-1}\right)$ & $\mathrm{C} /\left(\rho_{0} H\right)$ & $\lambda\left(\mathrm{W} \mathrm{m}^{-2} \mathrm{~K}^{-1}\right)$ & $1,004 C$ \\
$k_{d}\left(\mathrm{~s}^{-1}\right)$ & $(\mathrm{gC}) /(\Delta p)$ & $R\left(\mathrm{~J} \mathrm{~kg}^{-1} \mathrm{~K}^{-1}\right)$ & 287 \\
$k_{d^{\prime}}\left(\mathrm{s}^{-1}\right)$ & $(g C) /(\Delta p)$ & $\sigma\left(\mathrm{J} \mathrm{kg}^{-1} \mathrm{~Pa}^{-2}\right)$ & $2.16 \times 10^{-6}$ \\
$C\left(\mathrm{~kg} \mathrm{~m}^{-2} \mathrm{~s}^{-1}\right)$ & 0.015 & & \\
\hline
\end{tabular}

static stability $\sigma=-(R / p)\left(\partial T_{\mathrm{a}} / \partial p-1 /\left(\rho_{\mathrm{a}} c_{p}\right)\right)$, with $\rho_{a}$ the air density and $c_{p}$ the specific heat at constant pressure, is taken here to be a constant.

Note also that, thanks to the hydrostatic relation in pressure coordinates and the ideal gas relation $p=\rho_{\mathrm{a}} R T_{\mathrm{a}}$, the atmospheric temperature can be written as $T_{a}=-(p / R) f_{0}\left(\partial \psi_{\mathrm{a}} / \partial p\right)$. Since we are dealing here with a two-layer atmosphere, the temperature $T_{a}$ can simply be expressed, in terms of the baroclinic streamfunction $\left(\psi^{1}-\psi^{3}\right) / 2$, as $2 f_{0} / R \theta_{a}$. In the following, the field $\theta_{a}$ is also referred as the atmospheric temperature field.

For the ocean,

$$
\gamma_{o}\left(\frac{\partial T_{o}}{\partial t}+J\left(\psi_{o}, T_{o}\right)\right)=-\lambda\left(T_{o}-T_{a}\right)+E_{R},
$$

with

$$
E_{R}=-\sigma_{B} T_{o}^{4}+\epsilon_{a} \sigma_{B} T_{a}^{4}+R_{o} .
$$

Here, $R_{\mathrm{o}}$ is the shortwave radiation entering the ocean, and $\gamma_{\mathrm{o}}$ the heat capacity of the ocean.

The temperatures in both modules are linearized around a reference value to reduce the quartic terms of the energy balance Equations 7 and 9 to linear terms, assuming that the temperature fluctuations are small. This modification helps one to reduce the number of terms on the right-hand side of the ordinary differential Equations 6 and 8 when building the spectral low-order model.

The model fields in both its atmosphere and its ocean are developed in the Fourier series and truncated at a low order. The number of modes herein is fixed at 10 for the atmosphere and 8 for the ocean, leading to 20 ordinary differential equations for the former -10 for the barotropic streamfunction and 10 for the broclinic streamfunction-and 16 for the latter: 8 for the ocean streamfunction and 8 for the ocean temperature. This model configuration is the original VDDG one; see also Vannitsem (2017). The parameter values used in the present work are listed in Table 2.

In the VDDG model, there are a few key variables that display a large variability: those corresponding to the first mode $\sqrt{2} \cos \left(\pi y / L_{y}\right)$ of the Fourier series within the atmosphere, namely $\psi_{a, 1}$ and $\theta_{a, 1}$, are strongly affected by the radiative forcing, while the ocean variables associated with the Fourier mode $2 \sin \left(2 \pi y / L_{y}\right) \sin \left(\pi x / L_{x}\right)$ are strongly forced by the wind stress. Note that the latter mode is an idealized representation of the double-gyre structure often found in the dynamics of the upper layer of the world oceans (Dijkstra \& Ghil, 2005). These four variables are also crucial in the development of the LFV in the VDDG model (De Cruz et al., 2016; Vannitsem, 2017; Vannitsem et al., 2015) and will play a substantial role in the discussion of the results herein.

\subsection{Modeling the Tropical-Extratropical Interaction}

Schemm et al. (2018) investigated the changes of extratropical wintertime cyclogenesis when El Niño or La Niña events are occurring in the Tropical Pacific. These authors showed, in particular, that the background zonal-flow anomaly is more intense over the North Atlantic during La Niña, while it is stronger over the North Pacific during El Niño. This finding tells us that an important effect of the tropical forcing is to change the intensity of the zonal flow in either region, and that the impact of El Niño and La Niña differs from one region to the other.

To mimic this dynamic effect in the extratropical VDDG model used herein, we impose a direct linear forcing of the model's first barotropic atmospheric mode. It is this barotropic streamfunction mode that represents the intensity of the zonal flow within the atmosphere. Its dynamics is written as 


$$
\frac{d \psi_{a, 1}}{d t}=f_{1}\left(\psi_{a, 1}, \theta_{a, 1}\right)+g(X+Y)^{\prime}
$$

Here, $f_{1}\left(\psi_{a, 1}, \theta_{a, 1}\right)$ is the original right-hand side of the dynamical evolution (3a) of $\psi_{a, 1} ;(X+Y)^{\prime}$ represents the eastern Tropical Pacific basin's temperature anomalies, as given by Equation $1 \mathrm{~b}$ and plotted in Figure 1; and $g$ scales the intensity of the tropical forcing.

Thus $g$ represents the crucial forcing of the midlatitude VDDG model described in Section 2.2 above by the ENSO module of Section 2.1. In our setting, given a positive g-value, a positive, warm anomaly will induce an increase of $\psi_{a, 1}$, and hence of the first mode of the zonal barotropic flow, $-\psi_{a, 1} \partial\left(\sqrt{2} \cos \left(\pi y / L_{y}\right)\right) / \partial y$. This situation corresponds to the intensification of the zonal flow over the North Pacific during an El Niño. If, to the contrary, $g$ is negative, this would correspond to an intensification during La Niña that mimicks the ENSO effect over the North Atlantic.

The crude analogies with the impact of El Niño and La Niña on the atmosphere overlying the Atlantic and Pacific extratropical basins, along with the formulation of the ENSO forcing in our system, suggest that it is worth exploring both positive and negative $g$-values. Note that a similar analysis based on the western Tropical Pacific's temperature anomaly $y$ has been performed as well; its results were, in fact, quite similar to what is reported in this study.

\section{Dynamics of the ENSO-Forced Midlatitudes}

As explained in Section 2.3, the coupled ocean-atmosphere dynamics in our model's extratropical regions is forced by the Tropical Pacific. The impact of such a time-dependent forcing can be investigated in a self-consistent manner based on the concepts and tools of NDS theory and its PBAs. The latter are asymptotically invariant sets associated with a unique time-dependent forcing of a long-lived system that is started in the distant past; see Ghil et al. (2008, Appendix A) for a didactic presentation in the RDS case and Caraballo and Han (2017) for an accessible approach to both the NDS and RDS cases.

The concept of pullback attraction and the formal definition of a PBA in the deterministic, finite-dimensional case are introduced and motivated succinctly in Appendix B. Unlike in the better-known, autonomous setting, PBAs are themselves time-dependent objects. Interestingly, a unique global PBA can contain multiple local PBAs, as studied in the case of a double-gyre model of the wind-driven ocean circulation by Pierini et al. $(2016,2018)$. This novel type of multimodality will be illustrated in the present paper by the coexistence of two chaotic PBAs in our coupled ocean-atmosphere model.

In the present study, we compute the Lyapunov exponents of the ENSO-forced VDDG model as a key tool in the systematic investigation of its PBAs. More precisely, we compute the tangent linear system of the VDDG model along the forced trajectory. The Lyapunov exponents are then computed in this tangent space of 36 variables; see Appendix A for the definition of the Lyapunov exponents and for details on their calculation. The methodology follows Kuptsov and Parlitz (2012) but along a forced trajectory. Still, Ruelle (1984) showed that the Lyapunov exponents can still be computed and are unique, even in a dynamic system with time-dependent forcing, subject to the uniqueness and ergodicity conditions on the latter, which are verified in the present setting; see Appendix B for the details of this argument.

We performed a large number of ENSO-forced integrations of the VDDG model starting from different initial states in the extratropics. The length of the integrations was adapted to first explore a substantial number of parameter values with shorter runs and then successively increase the length of the runs to refine the analysis of carefully selected situations.

A long interval of $10,000,000$ time units $\simeq 3,072$ years was used for specific forcing and coupling parameter values to ensure convergence of the Lyapunov spectra. Some very long runs of 50,000,000 time units $\simeq 15,360$ years were used for two integrations investigating the robustness of the two PBAs found with shorter runs. In general, the transients of all these runs were taken equal to 10,000,000 time units $\simeq 3,072$ years. To check the convergence of trajectories toward the PBAs, we used runs that were 3,072-year long and had shorter transients of 1,536 years. 


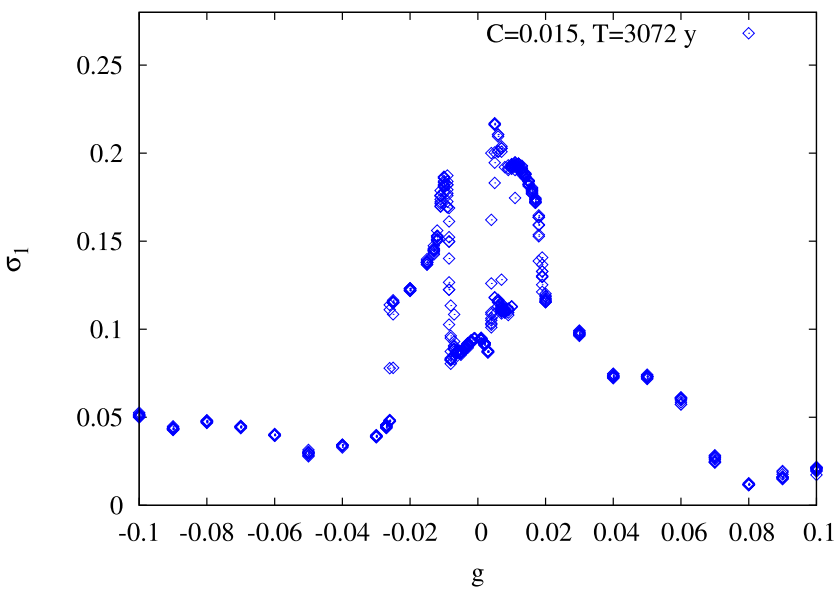

Figure 2. Leading Lyapunov exponent $\sigma_{1}$ (blue diamonds) as a function of the ENSO forcing parameter $g$ for periodic ENSO forcing as in Figure 1a. The air-sea coupling coefficient is $C=0.015$ and the analysis interval is $T=3,072$ years. For this $C$-value, a complex dependence emerges, with the possibility of multiple pullback attractors (PBAs) coexisting. ENSO, El Niño-Southern Oscillation.

\subsection{Periodic Forcing}

\subsubsection{The Leading Lyapunov Exponents}

Figure 2 displays the value of the leading Lyapunov exponent $\sigma_{1}$ as a function of the parameter $g$ when the tropical forcing is periodic in time, as in Figure 1a. The values plotted are for the configuration of the VDDG model that appears in Table 2, namely for $C=0.015$. For each forcing parameter $g, 10$ different initial conditions were selected. Note that, for certain ranges of $g$, several distinct values of the dominant Lyapunov exponent are obtained for different initial states in the remote past.

We have also performed an analysis along the same lines described below by using a value of the coupling coefficient of $C=0.008$ that equals roughly a half of the one finally retained. Since the ENSO forcing acts directly on the atmosphere alone, we expect its effects to be weaker on the coupled VDDG model the smaller the air-sea coupling $C$, which passes this forcing on to the ocean as well. The results obtained with $C=0.008$ (not shown) confirm this expectation inasmuch as they indicate a less rich dynamics, with a steady decrease of the amplitude of the dominant Lyapunov exponent as a function of the ENSO-forcing amplitude $g$.

For $C=0.015$, the behavior of the leading Lyapunov exponent is fairly smooth away from the interval -0.03 $\lesssim g \lesssim+0.02$, namely $\sigma_{1}$ is roughly constant for $-0.1 \leq g \lesssim-0.03$ and it is almost monotonically decreasing with increasing $g$ over the interval $+0.02 \lesssim g \leq+0.10$. This monotone behavior agrees with the heuristic idea that an increase in the tropical forcing makes the midlatitudes more predictable.

Near $g=0$, that is, for almost no ENSO forcing, $\sigma_{1}$ is of the order of 0.1 day $^{-1}$. Apparent discontinuities in $\sigma_{1}$, though, arise at the four $\sigma_{1}$-values of $\sigma_{1} \simeq-0.03,-0.005,+0.01$ and +0.02 . These jumps in the $\sigma_{1}$-values can be most easily understood by positing that there are two branches $\sigma_{1}^{ \pm}(g)$ of $\sigma_{1}$-values for $C=0.015$. The presence of two such branches suggests, in turn, the coexistence in this case of two distinct PBAs of the coupled VDDG model, as found in the purely oceanic double-gyre problem by Pierini et al. (2016), Pierini et al. (2018), and Pierini and Ghil (2021). This possibility is further discussed from the general perspective of NDS theory in Appendix B.

The transitions between these two local PBAs coexisting in the VDDG model's global PBA may be the generalization to this much more complex case of back-to-back saddle-node bifurcations in the autonomous setting described further in Appendix A; see also Ghil and Lucarini (2020, Section III.A and Figure 21) and references therein. Note that, over the two smaller intervals $-0.03 \lesssim \sigma_{1} \lesssim-0.005$ and $+0.01 \lesssim \sigma_{1} \lesssim+0.02 \sigma_{1}$ still decreases as the absolute values of $g$ increase away from 0 . To summarize, for $C=0.015$, the potential coexistence of two PBAs seems to extend over the interval $-0.03 \lesssim g \lesssim+0.02$, while it is entirely absent for $C=0.008$ (not shown).

Note that the ratio of the $\sigma_{1}$-values between the two branches in Figure 2 is of the order of 2 . The difference between the two PBAs is thus quite significant in terms of the potential predictability of midlatitude flow for the same Tropical Pacific surface temperature anomalies.

The discovery of multiple PBAs is not a trivial issue at all, as it most often has to rely on numerical experiments. Here, the significant difference in predictability properties implies that there are indeed two distinct PBAs that are both chaotic. There could be more such distinct PBAs, but we were not able to detect any additional ones. A more systematic analysis of how the bifurcation structure of the original VDDG model is affected by the periodic forcing could be a way to identify the emergence of several PBAs. Still, even if we were able to relate some PBAs to the local bifurcation structure of the autonomous system, one cannot preclude the possibility of hidden $P B A s$, like the hidden attractors in the autonomous case described by Leonov et al. (2015). 

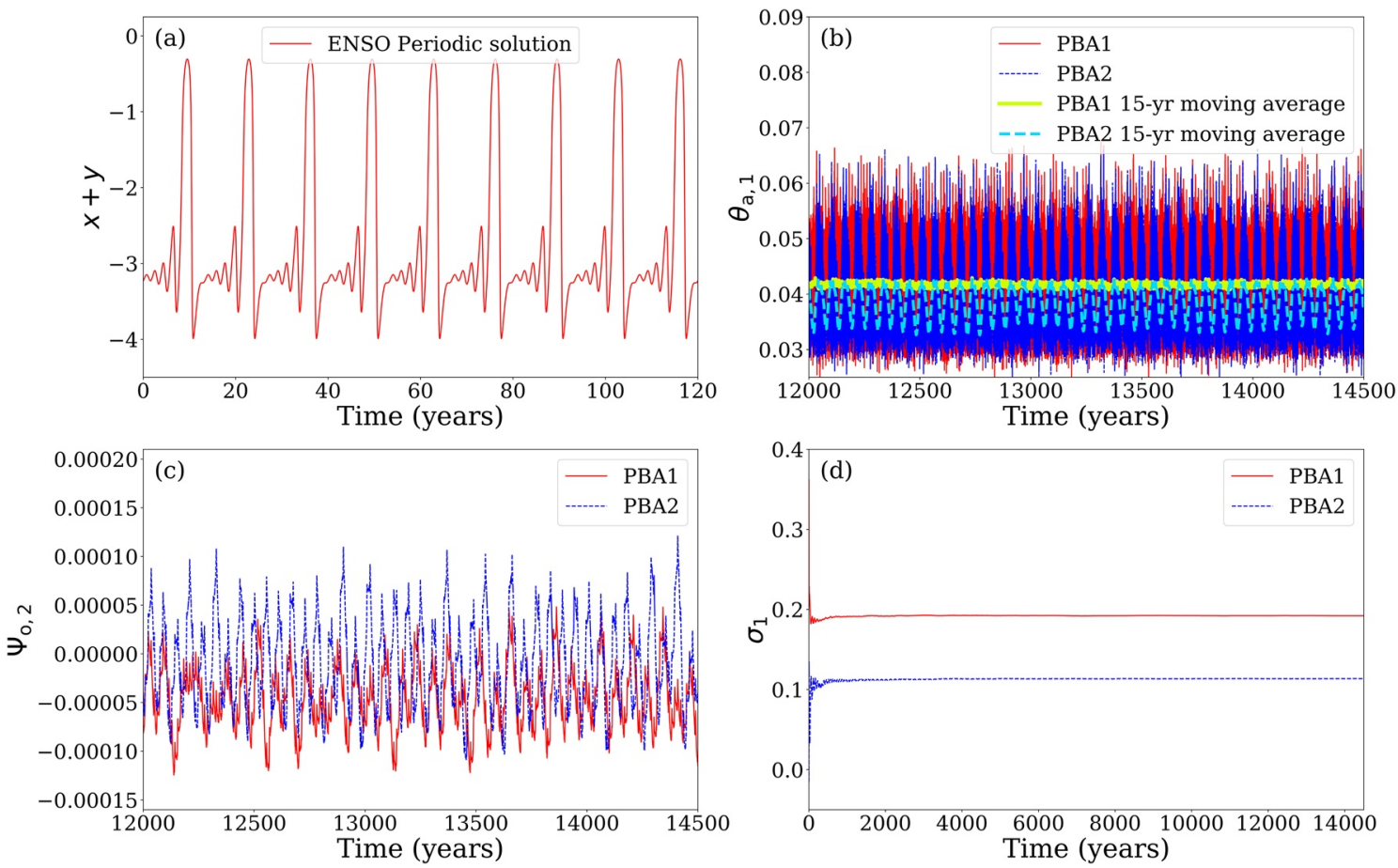

Figure 3. Model trajectory segments from a very long integration of roughly 15,000 years, using ENSO forcing that is periodic, with $g=0.01$ and air-sea coupling of $C=0.015$. (a) A segment of the ENSO forcing, identical to the one displayed in Figure 1a; (b) evolution of the atmospheric temperature variable $\theta_{a, 1}$; (c) evolution of the oceanic streamfunction variable $\psi_{o, 2}$; and (d) convergence of the two leading Lyapunov exponents $\sigma_{1}^{ \pm}$. In panels (b-d), the two curves, red and blue, correspond to the two PBAs obtained; in (b) the two additional curves correspond to 15 years moving averages, neon yellow for PBA1 and neon blue for PBA2. Cyclostationarity holds, given the periodic forcing, and any PBA segment (not shown) displays features similar to the ones in this figure. ENSO, El Niño-Southern Oscillation; PBA, pullback attractor.

The sharp transitions between the upper branch with $\sigma_{1}^{+}(g)$ to the lower branch with $\sigma_{1}^{-}(g)$ near $g \simeq-0.01$ and back near $g \simeq+0.01$ suggest that the upper branch continues to exist in the interval $-0.01 \lesssim g \lesssim+0.01$ as a "ghost PBA" that is no longer attained by forward integrations of the model. For autonomous systems in the atmospheric sciences, such "ghost equilibria" were defined by Legras and Ghil (1985) and "ghost limit cycles" by Kimoto and Ghil (1993). In these two cases, the exact meaning was clear: "A ghost fixed point is a fixed point that has become unstable in one or a few directions in phase space. Still, the system's trajectories will linger near it for extended time intervals. Likewise, a ghost limit cycle is a closed orbit that has become slightly unstable but is visited, again and again, by the system's trajectories.”; see Ghil et al. (2002).

In the present, nonautonomous case, it is less clear how a ghost PBA that loses its attractivity in one or more directions might behave and how model trajectories would continue to linger near it. In any case, the results illustrated in Figure 2 suggest some form of hysteresis between the upper and lower branches of PBAs in the case of $C=0.015$. These questions, while quite interesting, will require subsequent work to be solved.

\subsubsection{Typical Trajectories for $\mathrm{g}=\mathbf{0 . 0 1}$}

Let us now focus on the interesting case in which multiple PBAs are present at $g=0.01$. Figure 3 displays the solutions after a very long time integration of about 15,000 years, obtained using two distinct initial states that lead to distinct dominant exponents. Given the periodic forcing, asymptotic behavior is attained after a mere 3,072 years.

Panel (a) shows the temporal evolution of the eastern Tropical Pacific's temperature anomaly. The period of this ENSO forcing equals roughly 13 years. The maxima correspond to warm, El Niño events. In panel (b), the evolution of $\theta_{a, 1}(t)$, the first temperature mode in the baroclinic streamfunction (Vannitsem et al., 2015), is displayed for the two PBAs. 
The trajectory labeled PBA2 (blue curve) exhibits a clear sequence of low and high values that recur on long time scales of the order of 60 years. This oscillation is associated with the coupled ocean-atmosphere VDDG model's natural LFV in midlatitudes, which is similar to the one generated in the coupled model without ENSO forcing (Vannitsem et al., 2015). The trajectory labeled PBA1 (red curve), on the other hand, looks more erratic. A similar picture is found for the other atmospheric variables (not shown).

For the second mode $\psi_{o, 2}(t)$ of the ocean streamfunction displayed in panel (c), larger intensities of the ocean transport are found for PBA2 (blue curve), while a more erratic, albeit lower-amplitude evolution is found for PBA1 (red curve). Finally, in panel (d), the convergence of the leading Lyapunov exponents is shown, with the two attractors displaying distinct stability properties, as seen in Figure 2. Clearly, $\sigma_{1}^{ \pm}$correspond to PBAs 1 and 2, respectively, with the coupled PBA2 flow in the midlatitudes being stronger, as per panel (c), but more regular and stable, as per panel (d).

To further characterize the two local PBAs, we have performed a spectral analysis of the time series in Figure 3. The results are displayed in Figure $\mathrm{C} 1$ and Table $\mathrm{C} 1$, and they are described in greater detail in Appendix C1.

For PBA1, the periodicity $P_{E} \simeq 13$ years of the forcing dominates the atmospheric variables, while the ocean variables display a pronounced peak around 130 years; see Figures $\mathrm{C} 1 \mathrm{a}$ and $\mathrm{C} 1 \mathrm{~b}$. The signature of the periodic forcing is also present in the PBA1 spectrum for the ocean temperature mode $T_{o, 2}$. For PBA2, the atmospheric spectrum is dominated by the intrinsic variability of the VDDG model, namely $P_{V} \simeq 56$ years, while the oceanic variables only exhibit a notable very low-frequency peak at roughly 130 years.

\subsubsection{The Pullback Attractors for $\mathrm{g}=\mathbf{0 . 0 1}$}

To clarify the structure of the PBAs, 500 model integrations were carried out, each starting from a different initial state in the remote past. Snapshots are first displayed in Figure 4 at two different times (panels a and $\mathrm{b}$ ). The snapshots are projections onto the $\left(\theta_{a, 1}, T_{o, 2}\right)$ plane and colors indicate the size of the asymptotic Lyapunov exponent. The attractor PBA2 associated with the smaller values $\sigma_{1}^{-}$of the leading Lyapunov exponent (cold colors) has a much larger range of variability than PBA1 (warm colors). The projections of the two attractors onto the $\left(\theta_{a, 1}, T_{o, 2}\right)$ plane overlap substantially, as seen also in Figures $3 \mathrm{~b}$ and $3 \mathrm{c})$.

Due to the periodicity of the forcing, the attractors are expected to be cyclostationary and one can select key moments in the ENSO signal to get snapshots that are periodic, too. Panels (c and d) show these composite snapshots at the maximum and minimum of the periodic forcing in the eastern basin's temperature anomaly. These snapshots also illustrate the intricate structure of the two PBAs.

From the 500 trajectories produced, we also computed the histograms of the solution values as a function of time. These histograms are based on raw box count values and they are displayed in Figure 5 for PBA1 and in Figure 6 for PBA2. Here, PBA1 corresponds to the higher values of $\sigma_{1}$ and the smaller range of values in Figure 4, while PBA2 corresponds to the lower values of $\sigma_{1}$ and the larger range of values in Figure 4.

In Figure 6a, the impact of forcing on the distribution of possible values of $\psi_{a, 1}$ results in very large and sharp peaks in the zonal flow $\psi_{a, 1}$ when El Niño is strong. For the first mode $\theta_{a, 1}$ of atmospheric temperature (or of the baroclinic streamfunction), the peaks in panel (b) of the distribution are less pronounced but still significant; they are associated with stronger meridional gradients of the atmospheric temperature when El Niño events are occurring.

A similar effect of warm events is present for $T_{0,2}$, the second mode of midlatitude ocean temperature, displayed in Figure $6 \mathrm{~d}$. Note that, for this variable, the nondimensional values have a range of $0.05 \leq T_{o, 2} \leq 0.20$. This range corresponds roughly to dimensional temperature variations of $5-20^{\circ} \mathrm{C}$, which is quite reasonable.

For the ocean transport $\psi_{o, 2}$ in Figure $6 \mathrm{c}$, the range of values is also large and higher $H$-values occur near the most negative values. The impact of the forcing on this variable is also visible in the color shading variations of $\psi_{o, 2}$ distribution within the range $-0.00010 \leq \psi_{o, 2} \leq 0.0$. The total nondimensional range of the streamfunction is $-0.00015 \leq \psi_{o, 2} \leq+0.00020$, which corresponds to roughly -40 to $+50 \mathrm{~m}^{2} \mathrm{~s}^{-1}$ or to a velocity of -0.025 to $+0.030 \mathrm{~m} \mathrm{~s}^{-1}$. 

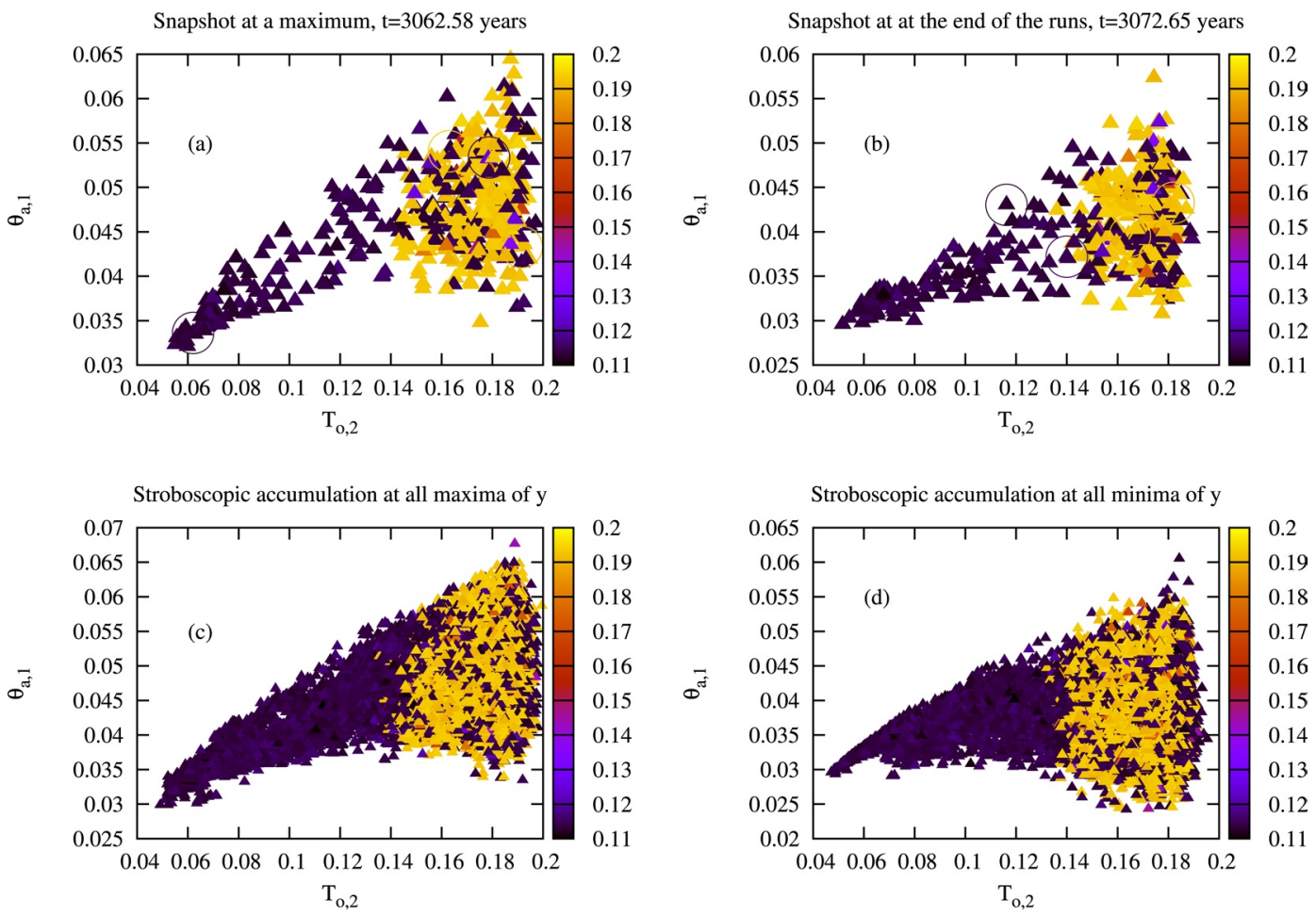

Figure 4. Coupled VDDG model snapshots obtained with 500 trajectories after a transient interval of 3,072 years (a and b) Individual snapshots at two different times of the periodic cycle, namely for 3,062.58 years and for $t=3,072.65$ years, which correspond to $X+Y=(X+Y)_{\max }$ and to the end of the run respectively; (c and d) the composites of instantaneous values at (c) the maxima and (d) the minima of the ENSO signal $X+Y$, given by Equation 1. The warm-colored and cold-colored solid triangles represent instantaneous maps of solutions belonging to PBA1 and PBA2, respectively, and they are associated with high or low values of $\sigma_{1}$; the color bar is for $\sigma_{1}$. The circles displayed in panels ( $\mathrm{a}$ and $\mathrm{b}$ ) locate the solutions that will be perturbed to check the stability of the PBAs. ENSO, El Niño-Southern Oscillation; PBA, pullback attractor.

The box count values in Figures $5 \mathrm{a}$ and $5 \mathrm{~b}$ of the atmospheric zonal flow $\psi_{a, 1}$ and temperature gradients $\theta_{a, 1}$ for PBA1 are not that different from those found in Figures $6 a$ and $6 \mathrm{~b}$ for PBA2. The main difference between the characteristics of PBA1 and those of PBA2 is the much larger range of high box count values (warm colors) of the ocean variables $\psi_{o, 2}$ and $T_{o, 2}$ in Figure $5 \mathrm{c}$ and especially in Figure $5 \mathrm{~d}$.

In Figure 6d, the highest $H$-values are concentrated very close to the most positive values of the temperature variable $T_{0,2}$, while in Figure 5d they extend pretty much across the entire range of temperature values; in other words, there is much more mixing in PBA1's ocean than in PBA2. On the other hand, Figure 6d shows higher-amplitude strands of solutions oscillating regularly between the highest and lowest temperature values. We shall return to this bunching of trajectories into oscillatory strands in the case of chaotic forcing in Section 3.2 below.

Figure 7 is a plot of the histograms of the local values of the leading Lyapunov exponents for both PBAs. For PBA2, the most frequently occurring values are located around 0 , and the periodic forcing is imposing a reduction of the higher values when strong El Niños are occurring. For PBA1, a similar feature is found, except that the most frequently occurring values cluster around $\sigma_{1} \simeq 0.25$.

Up to now, the results suggest that two different chaotic PBAs coexist in phase space at the forcing parameter value of $g=0.01$. But a crucial question when dealing with multiple attractors is their stability, and the possibility of jumping from one PBA to the other when perturbations in the initial states are introduced. If a small perturbation may lead to a transition to the other PBA, intricate boundaries between their respective basins of attractions could be present. If so, predictions of a specific solution type may be strongly affected by the presence nearby of solutions with very different properties. 

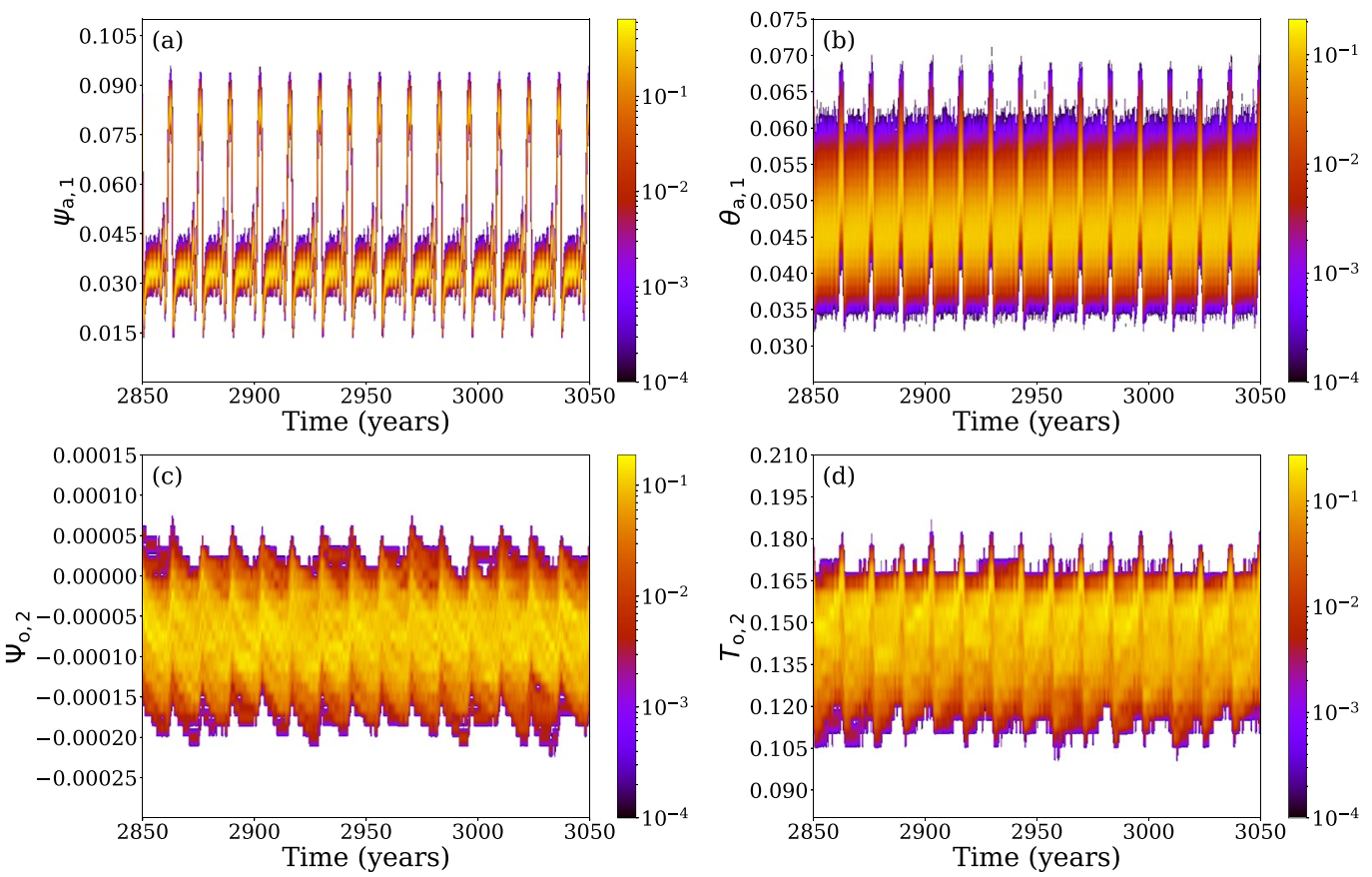

Figure 5. Solution histograms $H(t)$ as a function of time $t$ for 500 solutions obtained with periodic forcing and belonging to PBA1. The four histograms are for (a) $\psi_{a, 1}$; (b) $\theta_{a, 1}$; (c) $\psi_{o, 2}$; and (d) $T_{o, 2}$. The color bar indicates the (unnormalized) box count $H$ of the values on the $y$-axis. The histograms are smoothed and scaled logarithmically, that is, according to $\log _{10} H$, so as to get a better visual contrast. PBA, pullback attractor.

Such a possibility renders the problem of prediction - and in particular the ensemble predictions often carried out in the climate sciences-much harder, as some perfectly appropriate solutions could rapidly evolve far away from the system's real trajectory. Such rapid divergences could considerably affect the estimation
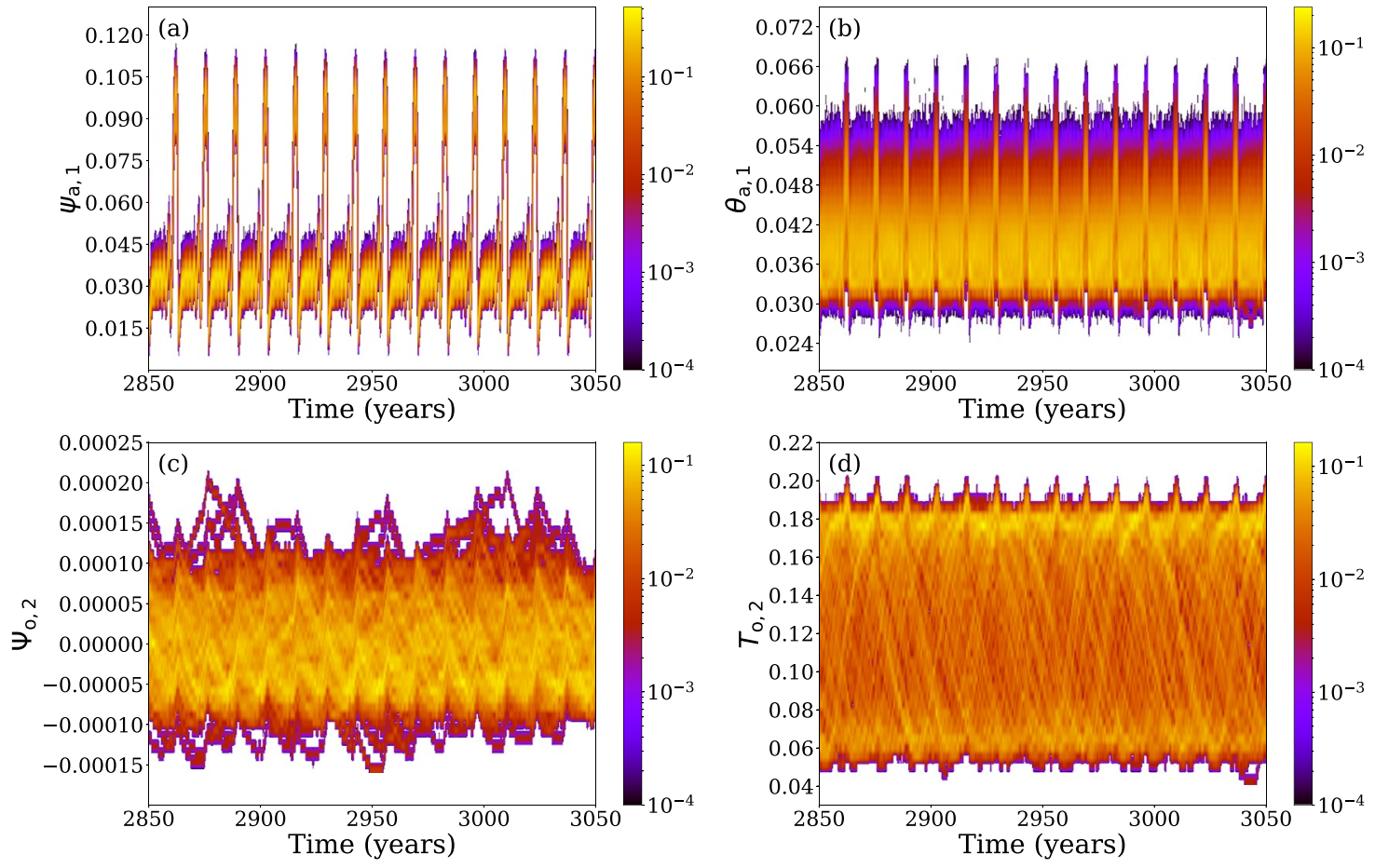

Figure 6. Same as Figure 5 but for PBA2. PBA, pullback attractor. 

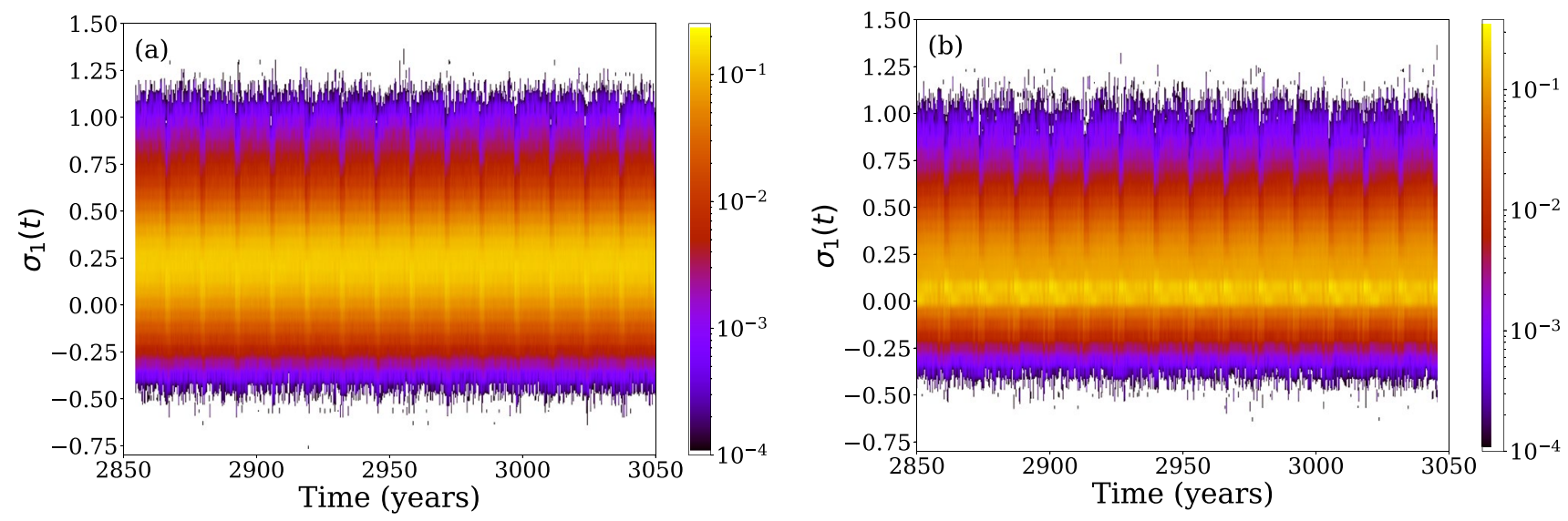

Figure 7. Time-evolving histogram of the local values of the leading Lyapunov exponent $\sigma_{1}$ of the two PBAs: (a) PBA1 and (b) PBA2. The local values are defined at each time step $\Delta t=0.05$ time units. Same scaling of the histograms as in Figures 5 and 6. PBA, pullback attractor.

of prediction errors. In the present case of periodic forcing, this situation will lead, in particular, to a loss of cycloergodicity, that is, temporal averages taken at multiples of the forcing period will no longer equal the ensemble averages.

To evaluate the stability of the two coexisting PBAs, four points in the snapshots of Figure $4 \mathrm{~b}$ were taken at random and perturbed with uniformly distributed random perturbations of amplitudes in the interval $\left[10^{-4}\right.$, $10^{-6}$ ]; each of the selected points is marked by a circle. The perturbations are introduced in all the variables, and the amplitude of $10^{-4}$ is of the same order at the natural variability of the ocean dynamics.

To assess the PBAs' stability, a new set of 1,536-year-long integrations are performed starting from these perturbed initial states and the convergence of the leading Lyapunov exponents $\sigma_{1}$ is analyzed during the second, 768-years-long part of these integrations. If a trajectory switches from one PBA to the other, then the corresponding $\sigma_{1}$ will change in value. As illustrated in Figure 8, there is no significant change in $\sigma_{1}$ with time, and hence no switching between the two PBAs.

Although this analysis is made for a few points only, the results suggest that the two PBAs are robust to changes of initial states, except for one large perturbation at point 3, in panel (c), which has the largest amplitude, of $10^{-4}$. The behavior of the two PBAs is quite similar when perturbing another four points within the snapshots of Figure 4a, chosen at the maximum of the ENSO forcing signal (not shown).

These results suggest that two rather robust chaotic PBAs coexist in the presence of periodic ENSO forcing. The two types of solutions are quite different. More specifically, for the forcing parameter value of $g=0.01$, there are key differences in their LFV: For PBA2, with its smaller $\sigma_{1}$, the LFV is dominated by multidecadal time scales, as in the LFV found by Vannitsem et al. (2015) for the VDDG model in the absence of ENSO forcing; this PBA also exhibits a high predictability, as expected. For PBA1, with its larger $\sigma_{1}$, the LFV has a much smaller range, it is closely related to the period of the external forcing, and it is characterized by a lower predictability.

\subsection{Chaotic Forcing}

\subsubsection{Typical Solutions}

We consider now the case of chaotic ENSO forcing upon the coupled VDDG midlatitude model obtained when the parameter values in the right column of Table 1 are used for the tropical module. The typical trajectory of this forcing is illustrated in Figure 9a. Note that, in this case, the recurrence times of large warm events are quite irregular and typically longer than when the forcing is periodic; compare Figures 1a and $1 \mathrm{~b}$.

For an ENSO forcing coefficient of $g=0.03$ and a coupling coefficient $C=0.015$, Figure 9 shows the typical evolution of two long integrations of about 15,000 years: one exhibits a higher value of the dominant Lyapunov exponent $\sigma_{1}$ (red curves) and the second one a lower value thereof (blue curves). The inequality 

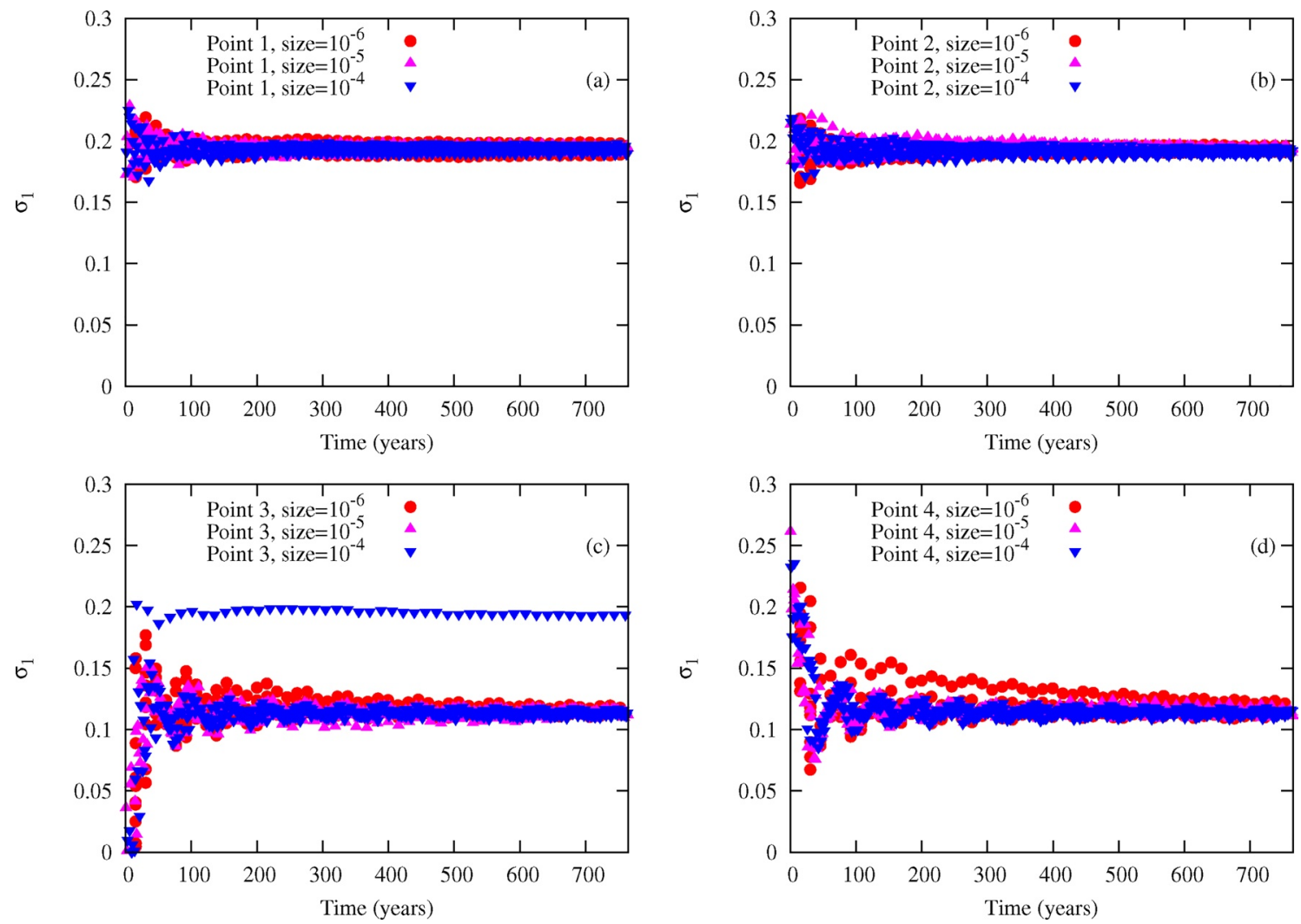

Figure 8. Convergence of $\sigma_{1}$ after perturbing the four points selected at random within the snapshots shown in Figure $4 \mathrm{~b}$, at the end of the 3,072-year-long trajectory analyzed therein. The legend in each of the four panels (a-d) indicates the amplitude of the perturbations: $10^{-6}$ (red), $10^{-5}$ (purple), and $10^{-4}$ (blue). For each amplitude, we performed 10 runs with 10 different random perturbations; in other words, 30 runs were done for each point.

$\sigma_{1}(\mathrm{PBA} 1)>\sigma_{1}(\mathrm{PBA} 2)$, observed already for the periodic case in Figure $3 \mathrm{~d}$ and is clearly confirmed in Figure $9 \mathrm{~d}$, where the convergence of $\sigma_{1}$ as a function of time is displayed for the chaotic forcing in panel (a). It is obvious, though, that the convergence in panel (d) here takes much longer than in Figure $3 \mathrm{~d}$ and is far from monotone.

Figures $9 \mathrm{~b}$ and $9 \mathrm{c}$ illustrate the corresponding evolution of the atmospheric variable $\theta_{a, 1}$ and the oceanic variable $\psi_{a, 1}$. In the two panels, the oscillations in both atmospheric and oceanic variables for PBA2 (blue curves) are associated with the slow dynamics on multidecadal time scales present already in the VDDG model with no ENSO forcing (Vannitsem et al., 2015). The ENSO forcing, though, seems to dominate the PBA1's LFV (red curves), in which the very slow intrinsic VDDG dynamics is not as strong. This difference between the two PBAs is particularly evident in the oceanic variable of panel (c).

The atmospheric oscillations in Figure 9b have large amplitudes for both PBAs throughout the time interval. For the oceanic oscillations in panel (c), though, the amplitude is quite intermittent, with long episodes of amplitudes that are quite small. Thus, the trajectory from PBA1 (red) has just a single large-amplitude burst, around 11,000 years.

An interesting feature is noticeable when taking a closer look at Figures $9 \mathrm{~b}$ and $9 \mathrm{c}$ : the mean value of the atmospheric temperatures $\theta_{a, 1}$ is lower during the high-amplitude episodes of the oceanic oscillations than during the low-amplitude episodes of the latter. The entries in Table 3 clearly show the very large amplitude difference between the ocean's leading variable $\psi_{o, 2}$, with an almost threefold factor between the quiescent middle episode (2) and the two much more active episodes (1) and (3)-6.8 $\times 10^{-5}$ versus $2.63 \times 10^{-4}$ and $2.35 \times 10^{-4}$. The corresponding difference in the means of the leading atmospheric variable $\theta_{a, 1}$ appears small at first-4.11 $\times 10^{-2}$ in the middle versus $3.85 \times 10^{-2}$ and $3.01 \times 10^{-2}$ at the two ends-but is highly significant given the very small standard deviations, of the order of $6 \times 10^{-3}$, for all three episodes. 

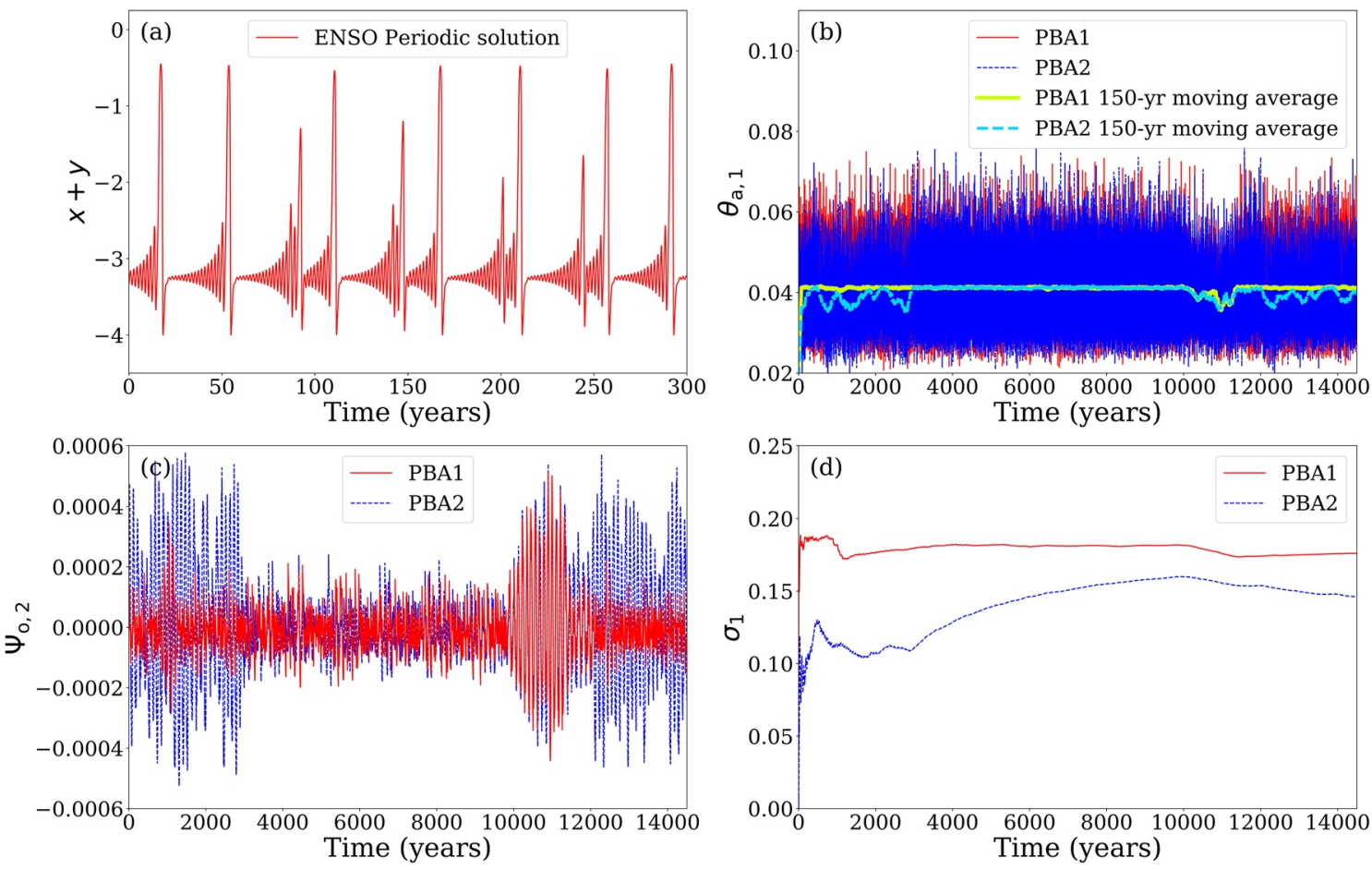

Figure 9. As in Figure 3 but for chaotic ENSO forcing, with $g=0.03$. (a) Time segment of 300 years from the chaotic forcing, identical to the one displayed in Figure 1b; (b and c) evolution of the variables $\theta_{a, 1}$ and $\psi_{o, 2}$ for a long integration of about 15,000 years, as in Figure 3, with the transient still fixed at 3,072 years and (d) convergence of the leading Lyapunov exponent over the same time interval. In panels (b-d), the curves for PBA1 are red and those for PBA2 are blue; in (b) the two additional curves correspond to 150-year moving averages, neon yellow for PBA1 and neon blue for PBA2. ENSO, El Niño-Southern Oscillation; PBA, pullback attractor.

Table 3

Means and Standard Deviations (std) of Atmospheric and Oceanic VDDG Variables for Chaotic ENSO Forcing

\begin{tabular}{lccc}
\hline Episode & Variable & Mean & Std \\
\hline$(1)$ & $\simeq 0-3,000$ years & & \\
& $\psi_{o, 2}$ & $2.43 \times 10^{-5}$ & $2.63 \times 10^{-4}$ \\
& $\theta_{a, 1}$ & $3.85 \times 10^{-2}$ & $6.55 \times 10^{-3}$ \\
$(2)$ & $\simeq 3,000-10,000$ years & & \\
& $\psi_{o, 2}$ & $-1.57 \times 10^{-5}$ & $6.8 \times 10^{-5}$ \\
& $\theta_{a, 1}$ & $4.11 \times 10^{-2}$ & $5.87 \times 10^{-3}$ \\
$(3)$ & $\simeq 10,000-15,000$ years & & \\
& $\psi_{o, 2}$ & $1.69 \times 10^{-5}$ & $2.35 \times 10^{-4}$ \\
& $\theta_{a, 1}$ & $3.91 \times 10^{-2}$ & $6.01 \times 10^{-3}$ \\
\hline
\end{tabular}

Notes. Results for atmospheric winds and oceanic currents in the PBA2 trajectory (blue) of Figure 9. The episodes $(1,2,3)$ are based on visual inspection of panel (c).
To better visualize these differences in the mean of the atmospheric variables, we have plotted in Figure $9 \mathrm{~b}$ the means of the atmospheric temperature $\theta_{a, 1}$ over a 150-year sliding window; they are indicated in neon yellow for the trajectory associated with PBA1 and in neon blue for the one associated with PBA2. It is clear from the figure that these moving averages are lower overall for PBA2 than for PBA1 during the initial and final episodes (1) and (3) in Table 3. More strikingly, they do exhibit significant fluctuations during these episodes for PBA2, which is not the case for PBA1.

The physical processes involved in the ocean-atmosphere coupling lead to enhanced oceanic variability suppressing the mean intensity of the meridional temperature gradient, and hence the intensity of the atmospheric westerly jet. In particular, an intensification of the oceanic circulation leads to an increase in the total heat toward the pole. The heat transported is rapidly exchanged with the atmosphere at high latitudes and thus leads to a reduction of the meridional temperature gradient within the atmosphere, which in turn reduces the westerly flow. On the contrary, when oceanic variability is low, poleward heat transport is low and the temperature gradients in both the ocean and the atmosphere are larger in the mean.

Given the strikingly intermittent behavior in Figure 9c, it is difficult to distinguish between the two PBAs, since transitions between the two basins of attractions do, apparently, occur. The difference in the behavior illustrated for very long trajectories in Figures 3 and 9 strongly suggests that the frequent transitions in the second case are induced by the chaotic ENSO forcing. 


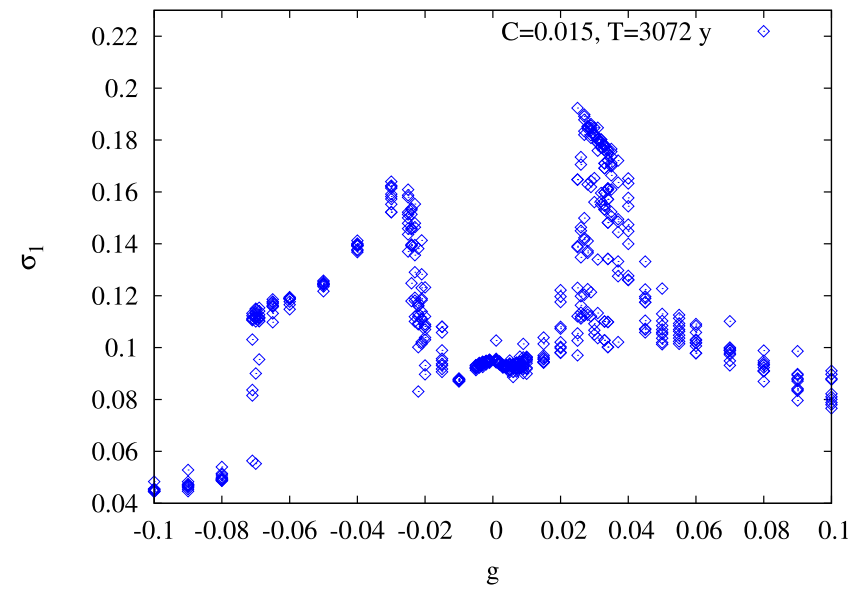

Figure 10. Same as Figure 2 but for the chaotic ENSO forcing, with the friction coefficient $C=0.015 .10$ long trajectories were obtained for each $g$-value, each with a 3,072-year transient and a 3,072-year analysis interval of the leading Lyapunov exponents $\sigma_{1}$. The complex dependence of the $\sigma_{1}$-values as a function of the ENSO forcing coefficient $g$ strongly suggests the coexistence of two local PBAs, as in the periodic-forcing case. ENSO, El Niño-Southern Oscillation; PBA, pullback attractor.
To better identify and characterize the two PBAs in this chaotic-forcing case, we performed the same type of spectral analysis as for the periodic-forcing case. The results are reported in Figure C2 and Table C1. As transitions between the two PBAs are occurring, the distinction between the two is less clear than in Figure C1.

The atmospheric variables of both PBAs are dominated by peaks at $P_{V} \simeq 56$ years and its first harmonic, while the corresponding oceanic variables have the former but not the latter. In addition, there is also a 130-year peak in PBA2's oceanic variables. Clearly, in the presence of chaotic forcing, it is the VDDG model intrinsic variability that plays the major role.

\subsubsection{The Leading Lyapunov Exponents}

The Lyapunov exponent computation used trajectories of $2 \times 3,072$ years in length, the first part being a transient and the second part being used to actually compute the leading Lyapunov exponents. Ten different initial conditions are used for each value of $g$. The results are shown in Figure 10.

Two coexistent PBAs and possible transitions between them are noticeable for a substantial range of forcing parameter values, namely $-0.07 \lesssim$ $g \lesssim 0.04$ and, in particular, $0.02 \lesssim g \lesssim 0.04$. The behavior here recalls what we found over the interval $-0.03 \lesssim g \lesssim+0.02$ for the periodic forcing illustrated in Figure 2 and discussed in Section 3.1.1, only more so, if one may say. The four jumps in $\sigma_{1}$ found here at $\mathrm{g} \simeq-0.07,-0.02,+0.03$, and +0.04 correspond to those found in Figure 2 at $g \simeq-0.03,-0.005,+$ 0.01 , and +0.02 .

The value of $\sigma_{1}$ near zero forcing, $g \simeq 0$, is still about $\sigma_{1} \simeq 0.1$ day $^{-1}$, as expected. But the chaotic forcing leads to a greater extent of the interval of PBA multiplicity, with $-0.07<-0.03$ and $0.02<0.04$ at both ends of the interval, as well as to a much greater scatter of the $\sigma_{1}$-values, especially all over the subinterval $0.02 \lesssim g \lesssim 0.04$. All this while the size of the discontinuity in the leading Lyapunov exponent is practically identical to the factor of 2 found in Figure 2, and the qualitative shape of the $\sigma_{1}=\sigma_{1}(\mathrm{~g})$ dependence in the two figures is highly similar. Hence, the greater scatter of $\sigma_{1}$-values that lie between $0.02 \lesssim g \lesssim 0.04$ and between 0.1 day $^{-1} \lesssim \sigma_{1} \lesssim 0.2$ day $^{-1}$ strongly suggests a larger number of transitions between the two local PBAs associated with $\sigma_{1}^{ \pm}$.

\subsubsection{Multivariate Singular Spectrum Analysis of the Local PBAs}

We turn now to the histograms of the variables as a function of time for an ensemble of 500 trajectories of $2 \times 3,072$ years. Since transitions appear to be possible between the two attractor basins-which are characterized by the presence or absence of multidecadal LFV-it is not easy to isolate the PBAs. We choose, therefore, a simple, scalar criterion: if the asymptotic value of the leading Lyapunov exponent $\sigma_{1}$ is larger than 0.14 , the trajectory is assumed to belong to PBA1, while if $\sigma_{1}<0.14$ we associate it with PBA2. Slight modifications of this threshold value do not modify the conclusions.

For PBA1, the modulation of the histograms by the forcing is quite striking in all four panels of Figure 11. In the case of the atmospheric variables of panels ( $a$ and $b$ ), the modulation appears to be cyclostationary, with a periodicity $P_{E}$ of roughly 35 years.

Since the ENSO forcing in Figures $1 \mathrm{~b}$ and $9 \mathrm{a}$ is quite irregular, we carried out a straightforward check of the ENSO signal produced by Equation 1 with the parameter values in the second column of Table 1. To do so, we first applied a data-adaptive filter provided by the multichannel singular spectrum analysis (M-SSA) of the ENSO model's three variables $(X, Y, Z)$, using $N=20,000$ data points, sampled every 56.075 days, with a window width of $M=2,000$ data points; thus $N / 3 \geq M \geq 35$ years, as recommended by Ghil et al. (2002), Alessio (2015), and references therein. Next, we carried out a maximum entropy spectral analysis of the SSA-filtered signal's $K=10$ leading reconstructed components (RCs: Ghil \& Vautard, 1991; Ghil et al., 2002), which capture $12 \%$ of the total variance. 


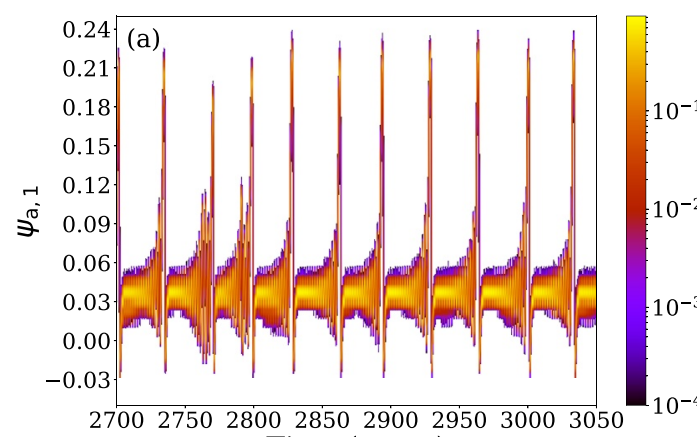

Time (years)

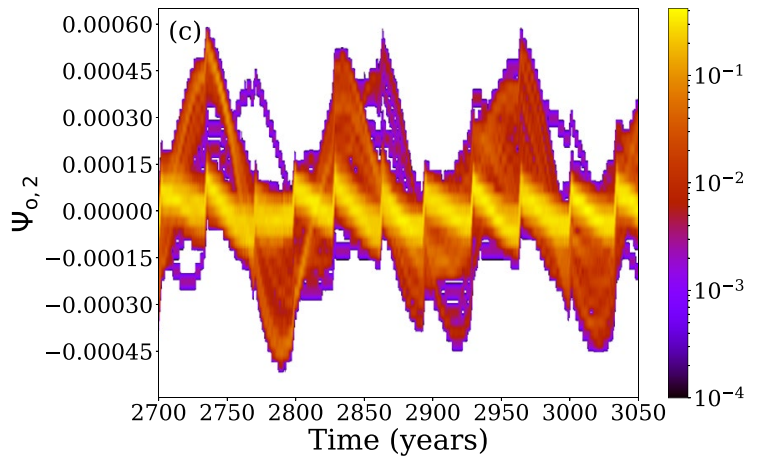

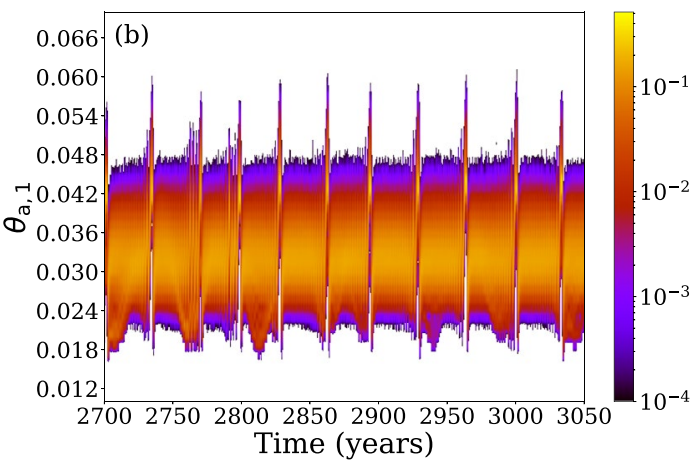

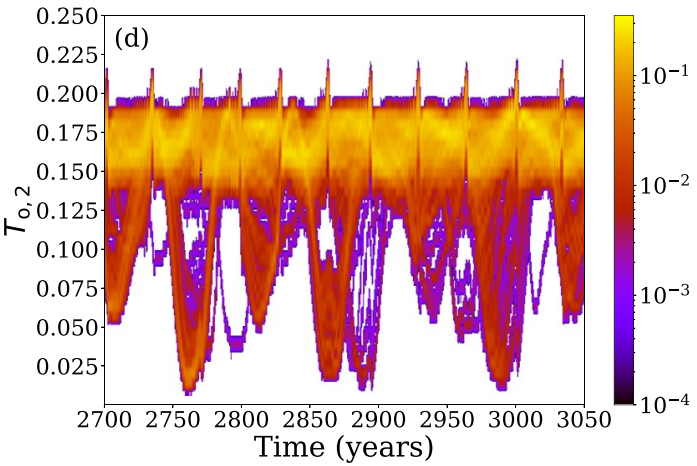

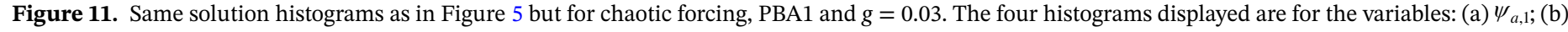

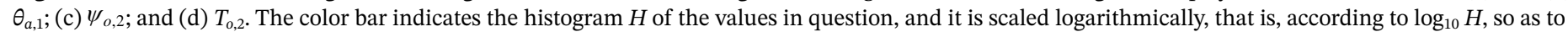
get a better visual contrast. PBA, pullback attractor.

As expected, the leading oscillatory mode of the signal, carried by the RC pair $(1,2)$ with a variance of $3 \%$ of the total, has a period of $P_{E}=36$ years. Subsequent pairs are associated with approximate harmonics of this basic signal: RCs $(3,4)$ form the third harmonic with period 11 years, RCs $(5,6)$ the second with period 17.7 years, $(7,8)$ has again the full period (34 years to be precise), and $(9,10)$ the fourth harmonic with 8.5 years. The highly visible irregularity of the ENSO forcing comes from the sum of all the remaining RCs. Thus, the VDDG model's atmospheric variables pick out the 36-years mode from the irregular ENSO forcing and amplify it, especially in the zonal velocity histogram of Figure 11a.

The interaction between the ENSO forcing and the midlatitude LFV is more complex for the oceanic variables in panels (c and d). In particular, the density maxima of the ocean temperatures $T_{o, 2}$ in panel (d) are shifted toward high values, as was the case for PBA2 and periodic forcing in Figure 5d. Moreover, both evolutive histograms of panels ( $c$ and $d$ ) are even more strongly affected by the ENSO forcing than the atmospheric histograms in Figures $11 \mathrm{a}$ and $11 \mathrm{~b}$. But the dominant periodicity $P_{V}$-seen most clearly in the warm colors-is of roughly 110 years.

To disentangle the complex effects of ENSO forcing on the midlatitude VDDG model's LFV, we carried out an M-SSA analysis of the latter's atmospheric and oceanic variables, separately. The trajectory used is 20,000 years long, with a forcing intensity of $g=0.03$, and it is taken from a trajectory associated with PBA2.

Results for the key Fourier modes of the VDDG model's atmospheric and oceanic modules appear in Table 4. The periodicities are obtained, as in the case of the ENSO signal, by maximum entropy spectral analysis of the SSA-filtered signal's $K=10$ leading RCs (Ghil et al., 2002; Alessio, 2015). As an additional verification of the results in the table, we also tested the individual time series of the atmospheric variable $\theta_{a, 1}$ and the oceanic variable $\psi_{o, 2}$. For $\theta_{a, 1}$ the 10 leading RCs capture $12 \%$ of the total variance and the peaks are at 39 , $15.7,11$, and 8.8 years; for $\psi_{a, 2}$, the partial variance captured is of $88 \%$ and the peaks are at 105 and 57 years.

Vannitsem et al (2015) found the dominant periodicity of the VDDG model in the absence of the time-dependent ENSO forcing to be roughly 60 years. Table 4 here confirms that, in this case, the dominant oscil- 
Table 4

Multichannel Singular Spectrum Analysis (M-SSA) of the Midlatitude Coupled VDDG Model, Subject to No Forcing $(g=0)$ or to Chaotic ENSO Forcing, With $g=0.03$; for Both Cases, the Internal-Coupling Parameter Value is $C=0.015$

\begin{tabular}{|c|c|c|c|}
\hline Forcing & Variables & $\mathrm{RC}$ pair [var\%] & Period (years) \\
\hline \multicolumn{4}{|l|}{ No } \\
\hline \multirow[t]{3}{*}{ [Atmos] } & $\left(\psi_{a, 1}, \theta_{a, 1}\right)$ & $(1,2)[70]$ & 58 \\
\hline & $\left(\psi_{a, 1}, \theta_{a, 1}\right)$ & $(3,6)[9]$ & 115 \\
\hline & $\left(\psi_{a, 1}, \theta_{a, 1}\right)$ & $(4,5)[8.5]$ & 38 \\
\hline \multirow[t]{3}{*}{ [Ocean] } & $\left(\psi_{o, 2}, T_{o, 2}\right)$ & $(1,2)[85]$ & 58 \\
\hline & $\left(\psi_{o, 2}, T_{o, 2}\right)$ & $(3,4)[8.5]$ & $38+115$ \\
\hline & $\left(\psi_{o, 2}, T_{o, 2}\right)$ & $(5,6)[5.2]$ & 115 \\
\hline \multicolumn{4}{|l|}{ Yes } \\
\hline \multirow[t]{5}{*}{ [Atmos] } & $\left(\psi_{a, 1}, \theta_{a, 1}\right)$ & $(1,2)[2.7]$ & 11 \\
\hline & $\left(\psi_{a, 1}, \theta_{a, 1}\right)$ & $(3,4)[2.5]$ & 35 \\
\hline & $\left(\psi_{a, 1}, \theta_{a, 1}\right)$ & $(5,6)[2.4]$ & 17 \\
\hline & $\left(\psi_{a, 1}, \theta_{a, 1}\right)$ & $(7,8)[2 . \%]$ & 34 \\
\hline & $\left(\psi_{a, 1}, \theta_{a, 1}\right)$ & $(9,10)[2.1]$ & 8.8 \\
\hline \multirow[t]{4}{*}{ [Ocean] } & $\left(\psi_{o, 2}, T_{o, 2}\right)$ & $(1,2)[55]$ & 54 \\
\hline & $\left(\psi_{o, 2}, T_{o, 2}\right)$ & $(3,4)[11]$ & $53+115$ \\
\hline & $\left(\psi_{o, 2}, T_{o, 2}\right)$ & $(5,8)[9.5]$ & Long trends \\
\hline & $\left(\psi_{o, 2}, T_{o, 2}\right)$ & $(6,7)[9.2]$ & 115 \\
\hline
\end{tabular}

Note. The length of the time series is $N=20,000$ years, the window width is $M=2,000$ years, and the time step is $\Delta t=56.075$ days. latory pair $(1,2)$ has a period of $P_{V} \simeq 58$ years; the associated variance is of $70 \%$ in the atmosphere and of $85 \%$ in the ocean. A shorter periodicity of 38 years and a longer one of 115 years are also present, in both the VDDG model's atmospheric and oceanic modules. The long, 115-year period is clearly a subharmonic of the main 58-year periodicity, since $58 \times$ $2=116$ years, and such small differences are negligible in the presence of complex nonlinear dynamics with a substantial fraction of continuous spectrum (Alessio, 2015; Ghil et al, 2002).

For chaotic ENSO forcing with scaling parameter $g=0.03$, as in Figures 9-11, M-SSA yields a leading RC pair $(1,2)$ with the period of 54 years in the ocean, where its variance is of $55 \%$. This period appears to be a slight modification-due to some rectification effect that remains to be clarified-of the VDDG model's intrinsic periodicity. The 54 years period is no longer present in the atmosphere, nor is this periodicity's long, 115-year subharmonic present in the latter.

As previously noted in Section 3.1.1, the ENSO forcing acts directly on the VDDG model's atmosphere and only indirectly on its ocean. Table 4 confirms that the periodicity $P_{E} \simeq 36$ years that dominates the chaotic ENSO forcing and was visually detected in Figures $11 \mathrm{a}$ and $11 \mathrm{~b}$ is indeed present in the M-SSA results. It appears in the table, as 35 years or 34 years in RC pairs $(3,4)$ and $(7.8)$, along with its second harmonic of 17 years in RCs $(5,6)$, its third harmonic of 11 years in RCs $(1,2)$, and as its fourth harmonic 8.8 years in RCs $(9,10)$. Given the highly anharmonic, spiky appearance of this signal in Figure 11a, the heavy loading of the harmonics is not surprising at all, with the variances of the dominant $P_{E}$ and its four harmonics capturing variances between $2.7 \%$ and $2.1 \%$.

The M-SSA results for the oceanic variables include only the intrinsic periodicity $P_{V}$, in RC pairs $(1,2)$ and $(3,4)$, along with its subharmonic of 115 years in pairs $(3,4)$ and $(6,7)$, and even longer trends in RCs 5 and 8 . The highly anharmonic and interwoven character of the oscillations makes their varimax separation still rather incomplete. The distinct spectral signatures of the atmospheric and oceanic variables in Table 4 were found also by Vannitsem and Ghil (2017) in observational data for coupled ocean-atmosphere dynamics over the North Atlantic basin.

The highly visible $P_{V} \simeq 115$-year periodicity in the oceanic histograms of Figures 11c and 11d is a degree-3 subharmonic of the ENSO forcing at roughly 36 years. It is the presence of this 110-year periodicity in the oceanic spectrum of the unforced VDDG model that causes the nonlinear resonance with the ENSO forcing periodicity of $P_{E} \simeq 36$ years to occur, rather than a simpler resonance with the forcing periodicity itself. Such phenomena have been found in paleoclimate studies (Ghil, 1994, and references therein) and will be discussed further in Section 4.2.

Figures 11c and 11d reveal another interesting phenomenon, namely the splitting of tracks between groups of trajectories that we called strands in discussing Figure 6d. This splitting is even more obvious in Figure 12 for PBA2, and we will describe it in discussing the latter figure below.

For PBA2, the picture in Figure 12 is quite different from the one for PBA1: a strong LFV signal on time scales of $P_{V} \simeq 110$ years is present in all the midlatitude variables, while the mean periodicity of the forcing is much shorter, of $P_{E} \simeq 35$ years. This feature is particularly visible when following the density maxima for the ocean variables in panels ( $c$ and d). It is quite intriguing that, in this evolutionary histogram representation, most of the trajectories fall into one of three distinct strands that seem to have the same very large periodicity but are phase shifted with respect to each other. The mutual phase shifts are by $\sim 35$ years, that, they roughly equal the periodicity of the forcing, and one clearly sees in panel (c) that $P_{V} \simeq 3 P_{E}$. 

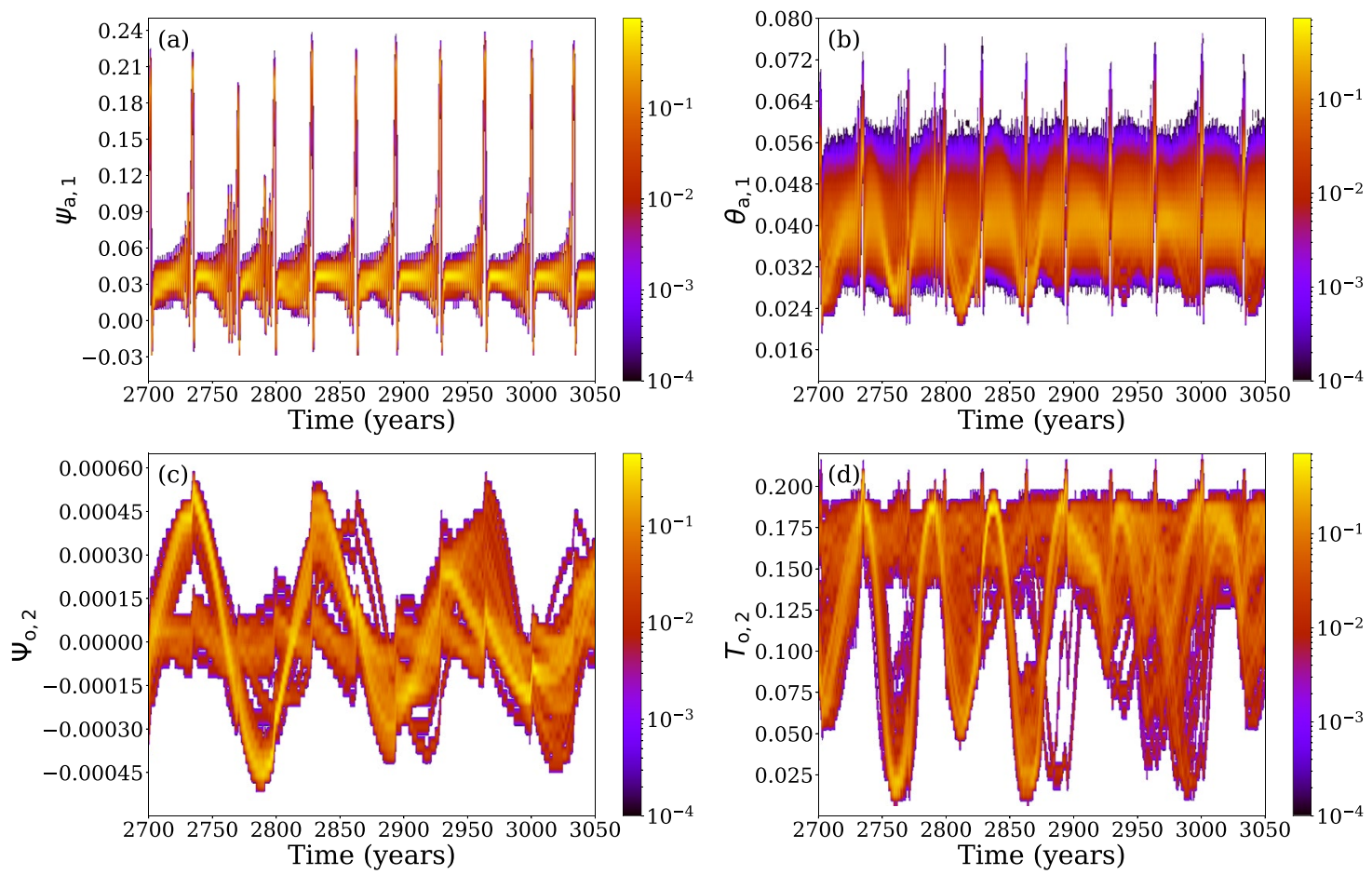

Figure 12. As in Figure 11, but for PBA2. PBA, pullback attractor.

This phenomenon indicates a more coherent dynamics induced by the presence of the forcing, even if the latter acts on a distinct, and much shorter, characteristic time scale. The striking interaction of intrinsic and forced behavior is also present in the variations of the local predictability for PBA2, as illustrated in Figure 13b: comparison with Figure 12d shows that high predictability occurs preferentially when low values of $T_{o, 2}$ predominate. This conditioning of high predictability on low $T_{o, 2}$ values for PBA2 contrasts with the increase of predictability for PBA1 in Figure 13a being synchronized with high amplitudes of the external forcing in Figure 12a. Further comments on synchronization by time-dependent forcing appear at the end of Section 4.1.

A key question is whether the statistic properties of the ENSO-forced VDDG dynamics illustrated in Figures 11-13 are robust and, in particular, independent of the time of initialization of the trajectories. To
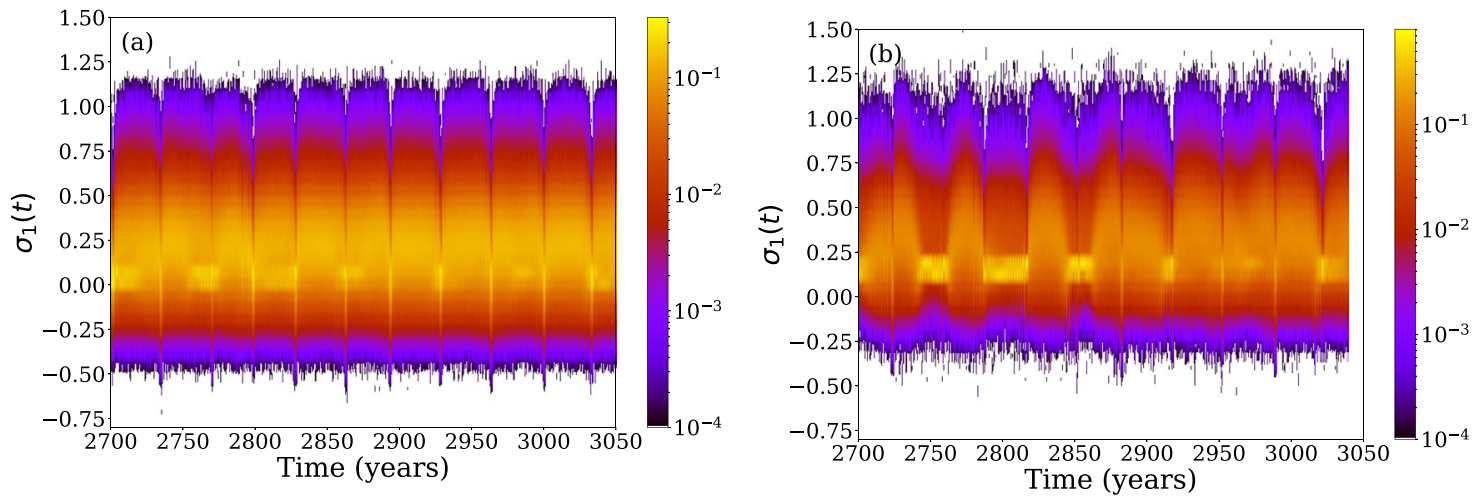

Figure 13. Same time-evolving histogram of $\sigma_{1}$ as in Figure 7 but for chaotic ENSO forcing with $g=0.03$ : (a) PBA1 and (b) PBA2. ENSO, El Niño-Southern Oscillation; PBA, pullback attractor. 

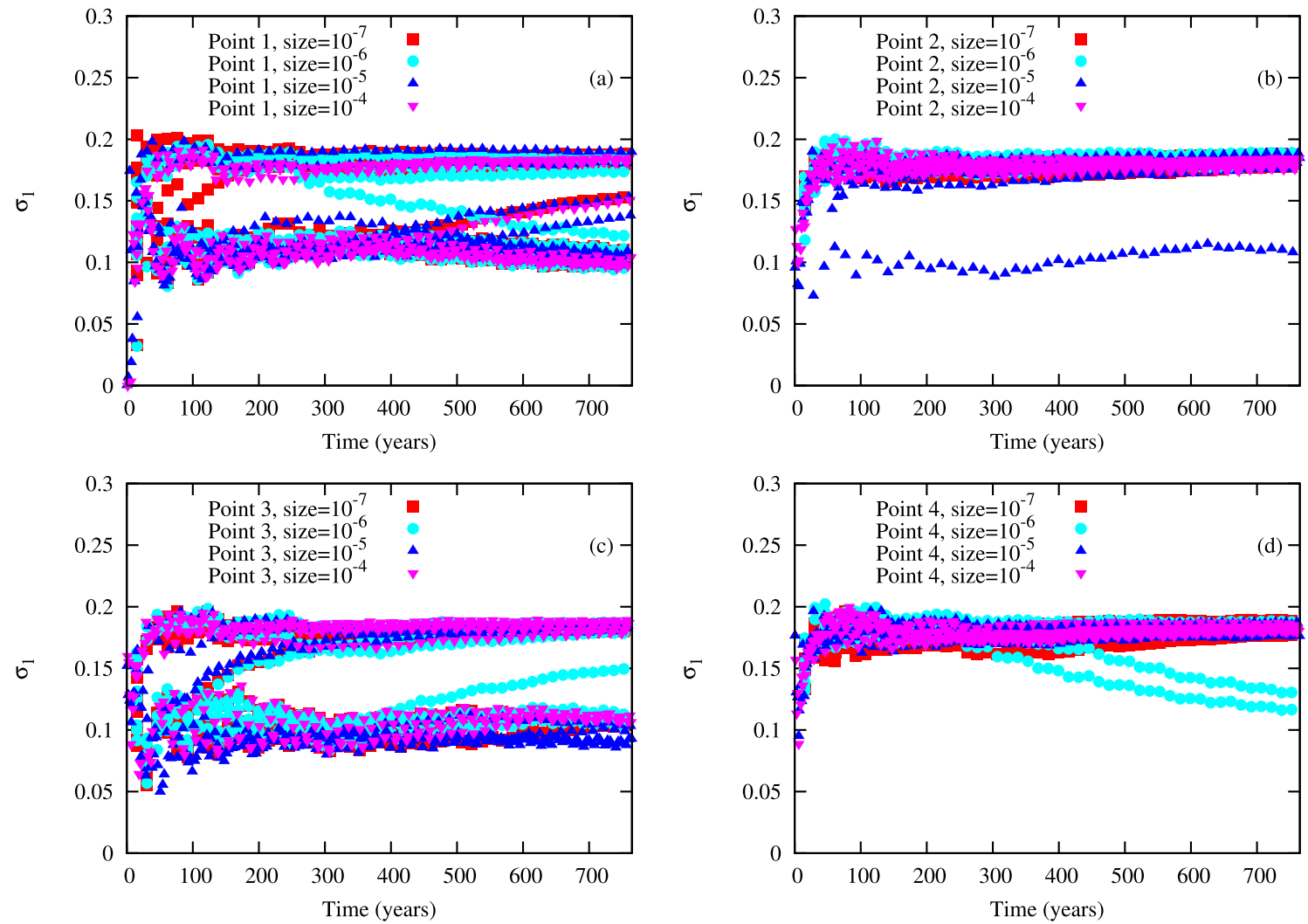

Figure 14. Convergence of the leading Lyapunov exponent $\sigma_{1}$ after perturbing the initial states of four different points selected at random on the two PBAs in the middle of the total analysis interval of $2 \times 3,072$ years. The convergence is analyzed over 768 years. For several points on PBA2, transitions to PBA1 are found. Panels $(a-d)$ show the results for the four randomly chosen initial states. PBA, pullback attractor.

check the latter issue, we started another set of 500 trajectories from a new set of initial states of the VDDG model, 1,536 years in the past.

The histograms are now displayed in Figures $\mathrm{C} 3$ and C4, for PBA1 and PBA2, respectively. Comparison with Figures 11 and 12 clearly shows that (a) the shorter transient of 1,536 years already provides sufficient convergence to either of the two PBAs' asymptotic behavior, respectively; and (b) that the evolution of the solutions within this asymptotic regime still separates into the same two PBAs and does not depend on the details of the sample set used for initialization.

The characteristics of each PBA are pairwise the same, and the only differences are clearly attributable to sampling issues. Hence, the distributions displayed in Figures 11-13 are genuine asymptotic features of our coupled VDDG model's solution set for the specific forcing chosen.

As for the periodic case, we may wonder whether the asymptotic dynamics we found is robust to perturbations of the state of the extratropical system. Several experiments have been performed with different perturbation amplitudes, as illustrated in Figure 14. The perturbations are introduced around four different points at two different instants in the forcing signal's evolution. Two of these points are associated with PBA1's high values of the dominant Lyapunov exponent $\sigma_{1}$ and illustrated in panels (a and c) of the figure, while the two others, illustrated in panels (b and d), are associated with PBA2 and a low value of $\sigma_{1}$.

Transitions are noticeable in both directions, from PBA1 to PBA2 and vice-versa, but PBA1 is clearly more robust than PBA2. Even for very small perturbations of the solutions, with amplitudes as low as $10^{-7}$, some solutions initially belonging to PBA2 switch to PBA1. The transitions from PBA1 to PBA2 seem to occur only for relatively large perturbations. It thus appears that, in spite of its larger $\sigma_{1}$, it is PBA1 that has the larger basin of attraction. 


\section{Concluding Remarks}

\subsection{Summary}

As stated in Section 1, the theory of nonautonomous dynamical systems (NDSs) and of their (PBAs provides key concepts and tools to study the dynamics of systems with time-dependent forcing and coefficients. In recent years, this theory has provided several important advances in the climate sciences, as reviewed, for instance, by Ghil and Lucarini (2020) and Tél et al. (2020).

In the present study, we have studied an intermediate climate model, developed by Vannitsem et al. (2015), with higher resolution and more physics than most models to which the concepts and tools of NDS theory and PBAs have been applied heretofore. This model was dubbed herein the VDDG model and summarized in Section 2. This coupled ocean-atmosphere model represents extratropical basin-wide dynamics and it comprises two reduced-order modules, atmospheric and oceanic. Vannitsem et al. (2015) found in the VDDG model LFV that develops coherently in both modules, while Vannitsem and Ghil (2017) verified that its multidecadal coupled mode shares common features with reanalysis data sets. The VDDG model was forced herein by an ENSO model based on the study of Jin (1996, 1997), An and Jin (2004), Timmermann et al. (2003), and Roberts et al. (2016).

The work carried out herein is clearly of an exploratory, numerical nature. Subject to the usual assumptions about the system's ergodicity or-as E. N. Lorenz preferred to address the issue, its total transitivity or almost intransitivity - we used systematically single model trajectories or subsets thereof to ascertain the validity of certain conjectures about the local PBAs, their coexistence, and the transitions between them. The length and number of these trajectories were chosen carefully, as were the methods for the study of their properties. Such care is necessary and can be reassuring but it does not replace rigorous mathematical proofs. Thus, all the inferences drawn are subject to further validation or invalidation.

For both periodic and chaotic ENSO forcing, the extratropical model displays multiple PBAs for a substantial range of the scaling parameter $g$. This result confirms that multiplicity of PBAs is not limited to highly idealized, low-order dynamics. For the two PBAs coexisting here, the LFV behavior can be drastically different: PBA2 features a strong multidecadal signal, intrinsic to the VDDG model, while for PBA1 a much weaker signal is dominated by the recurrence of warm El Niño and cold La Niña events; see Figures 3 and 9 and Sections 3.1.3, and 3.2, respectively.

This difference in the nature of the two PBAs' variability is accompanied by drastically different predictability properties, as shown by the respective leading Lyapunov exponents $\sigma_{1}$, with $\sigma_{1}^{+}$for PBA1 and $\sigma_{1}^{-}$for PBA2; roughly speaking, $\sigma_{1}^{+} \simeq 2 \sigma_{1}^{-}$, as seen in Figures 3 and $9 \mathrm{~d}$. This finding is consistent with the fact that, in the unforced VDDG model, predictability was higher when multidecadal LFV was present (Vannitsem et al., 2015). When the midlatitude model is forced by the chaotic ENSO solution, an even richer dynamics is found for PBA2, characterized by LFV on centennial time scales: as seen in Figure 12c, phase locking of all the model trajectories along one of three paths in phase space, or strands, occurs in this case, but not for PBA1.

In the present study, we have also explored the stability of these attractors by perturbing the initial states of the extratropical model and following the trajectories starting from the perturbed states. In the case of periodic ENSO forcing, the extratropical model looks quite robust even when the initial perturbations are of the same order of magnitude as the oceanic streamfunction field's variability; see Figures 8 and 14.

When the system is forced by the chaotic ENSO signal, though, PBA1 is much more robust than PBA2. The latter displays trajectories that can easily escape to the other attractor once perturbed, even for perturbations that are quite small in amplitude; see Figures 14a-14c. Thus PBA2 could be qualified as metastable. For larger initial-perturbation amplitudes, the perturbed solutions can visit one or the other attractor, as in Figure 14, suggesting that holes or melancholia states (Ghil \& Lucarini, 2020; Lucarini \& Bódai, 2017) may develop in the basin boundaries of the local PBAs.

An additional matter of both theoretical and practical interest in the present results is the complex interplay between time-dependent forcing and intrinsic climate variability in the spectral domain. Visual examination of Figures $6 c, 6 d, 11$, and 12 already suggested a complex interaction between the ENSO forcing and 
the VDDG model's intrinsic variability. In Section 3.2, we took a closer look at the spectral characteristics of the chaotic ENSO forcing, on the one hand, and on the VDDG's model's behavior with and without chaotic ENSO, on the other. Table 4, in particular, confirmed the results of Vannitsem et al. (2015) as to the VDDG model's dominant intrinsic periodicity of $P_{V} \simeq 58$ years and pointed to an important subharmonic thereof, at $2 \times P_{V} \simeq 115$ years.

In the presence of chaotic ENSO forcing, the VDDG atmospheric module does show the dominant periodicity of the forcing, at $P_{E} \simeq 36$ years, and several harmonics thereof. The latter is due to the highly anharmonic, spiky character of the ENSO signal itself, cf. Figure 1b, as well as to the model's rather noisy atmosphere, cf. Vannitsem et al. (2015).

The ENSO-forced VDDG model's ocean LFV, though, is still dominated by the intrinsic periodicity $P_{V} \simeq 58$ years, and by its subharmonic $2 \times P_{V} \simeq 115$ years. The latter is, at the same time, a subharmonic of the forcing frequency, $3 \times P_{E} \simeq 115$ years, and it is precisely this nonlinear resonance, $2 \times P_{V} \simeq 3 \times P_{E}$, that characterizes the interaction of the chaotic forcing with the model's chaotic LFV.

Such rational, as opposed to 1:1, resonances are well known in other subfields of the climate sciences. Thus the Great Inequality of Jupiter and Saturn — that is, the 2:5 near-resonance between the revolution periods of Jupiter and Saturn around the Sun (Wilson, 1985) — constitutes a major perturbation of the regular, quasi-periodic evolution of the solar system (Varadi et al., 1999). And the interaction of quasi-periodic, orbitally induced insolation forcing and of a still periodic, but nonlinear climate oscillator can lead to multiple rational resonances and combination tones, as well as to chaotic Quaternary glaciation cycles (Ghil, 1994). In the latter case, the intrinsic climate oscillations were due to coupling of an energy balance model with an ice mass balance model into a very low-order model (Ghil, 1994; Ghil \& Childress, 1987; Le Treut et al., 1988).

More generally, synchronization effects of periodic, random, or rapidly fluctuating deterministic forcing have been studied across the NDS and RDS literature (Young, 2017, and references therein). Related issues are discussed in Appendix B herein, as well as elsewhere in the climate sciences (Checkroun et al., 2011; De Saedeleer et al., 2013; Ghil et al., 2008).

\subsection{Discussion}

In the present analysis, we have focused merely on two different types of ENSO forcing, periodic and chaotic, and on two specific air-sea coupling parameter values that lead to the presence of multiple PBAs. At this stage, we have thus only lifted the veil on a small number of possible solutions and types of behavior in the current model. The striking results, though, warrant further exploration. Proceeding more systematically, even for the current model version, requires intensive computer calculations as the number of trajectories needed is large and the necessary run times are quite long.

This being said, the possibility of alternating between two PBAs with very different behavior and predictability-even in the presence of purely deterministic forcing, whether periodic or chaotic-has profound implications for interannual climate predictions and longer-term climate projections (e.g., IPCC, 2014). Lorenz (1990) already formulated a low-order, midlatitude atmospheric model with seasonal forcing, in which the bistability of a perpetual-summer climate, combined with the chaotic character of the unique perpetual-winter climate, led to low interannual predictability. In fact, the Lorenz (1990) model's two summer climates, which were both stable at constant forcing, were characterized, respectively, by a strong versus a weak oscillation of the model's westerly flow, somewhat like our PBA2 and PBA1 in the case of periodic ENSO forcing.

The present results thus extend those of Lorenz (1990) in two ways: (i) to longer, multidecadal time scales; and (ii) to a model that couples the atmosphere and ocean, as well as the Tropics and extratropics. These results confirm, therewith, the limitations on predictability due to the interactions of time-dependent forcing and intrinsic climate variability for a model that sits on a substantially higher rung of the climate modeling hierarchy; for the importance and usefulness of such a hierarchy, see, for instance, Ghil (2001), Held (2005), and Ghil and Lucarini (2020), and references therein.

It thus appears fairly likely that the climate system could possess-on the time scales of interest hereinmultiple PBAs that are affected by chaotic forcing. In the kind of situation depicted in Figure 14, individual 
members of a typical prediction ensemble will persist in a single local PBA or visit the two (or more) coexisting PBAs; hence, the first and second moments of the distribution generated by the ensemble are of very limited use for actual prediction. Since switches between PBAs may be highly intermittent, with very long intervals of dwelling in either one of them, more reliable ensemble predictions would require both much longer runs and many more of them.

This state of affairs also implies that analyzing the dynamics of a more detailed and, presumably, more realistic climate model requires a very large ensemble starting from initial states in the distant past. Achieving this computational feat would allow for as long a spin-up interval as necessary to reach the model's global attractor, as well as for a very long time to analyze possibly multiple local PBAs, as done herein. Such an achievement would also shed further light on the so-called signal-to-noise paradox in IPCC-class climate models.

For quite a while, there has been a suspicion that the LFV signal in such models is much weaker than observed (Kravtsov et al., 2018; Smith et al., 2020). This underestimation of interannual-to-multidecadal LFV is usually attributed to omission of mechanisms and errors in the parameter values used in model development. O'Reilly et al. (2019), though, have raised the possibility that the choice of stratospheric initial states might play a role in the limited amplitude of a detailed climate model's North Atlantic Oscillation. The results herein indicate that the limited, and possibly quite suboptimal, choice of initial states might play a key role in missing much of IPCC-class models' range of long-term behavior in general, and of vigorous LFV in particular.

The spectacular channeling of highly irregular trajectories into distinct strands in phase space, as found in panels (c and d) of Figures 12 and C4, illustrates the complex dynamics that the interaction of chaotic forcing with intrinsic LFV can give rise to, namely the phase locking by the former of the latter's set of trajectories along very specific paths. Other forms of striking response of chaotic variability to external forcing had been reported already in more highly idealized settings (Checkroun et al., 2011, 2018; Pierini et al., 2016, 2018; Pierini, 2020). As already mentioned in a previous paragraph of this section, the findings herein raise the likelihood of such interesting and challenging behavior occurring in the climate system itself and require, therefore, further confirmation by the study of increasingly detailed and sophisticated models.

As this discussion points out, there are substantial deficiencies left in high-end, IPCC-class models matching observations and reanalyzes, on the one hand, and a large gap between interesting behavior in reduced-order models and the high-end ones, on the other. Several review studies over the last two decades have emphasized, therefore, the need for model studies across a full hierarchy of models (e.g., Ghil, 2001, 2019; Ghil \& Lucarini, 2020; Held, 2005), as well as of more advanced types of analysis methods being applied systematically to both models and observations (e.g., Ghil et al., 2002; Groth et al., 2017).

The Modular Arbitrary-Order Ocean-Atmosphere Model (MAOOAM: De Cruz et al., 2016) is well adapted to studying the above-mentioned striking results across a hierarchy of increasingly well-resolved models, given its modular structure that facilitates the use of an arbitrary number of basis functions. Of course, this does not automatically include additional physical or chemical mechanisms that are present in high-end models. Still, performing multiannual climate predictions and multidecadal climate projections in the presence of large uncertainties in the external forcing, on the one side, and the limited amount of computer resources to run comprehensive climate models, on the other, pose great challenges. These challenges compel us to explore the wealth of nonlinear climate behavior in the presence of time-dependent forcing, natural and anthropogenic, across the intermediate rungs that MAOOAM can occupy within a full hierarchy of climate models.

\section{Appendix A: Dynamical Systems Tools: A Primer}

In this appendix, we provide a brief overview of the basic tools used in dynamical systems theory (e.g., Arnol'd, 2012; Guckenheimer \& Holmes, 1983; Nicolis, 1995). For the sake of simplicity, we assume that the system under consideration is described by a set of ordinary differential equations (ODEs), like the ones introduced in Sections 2.1 and 2.2. This set of ODEs can be formally written as 


$$
\frac{d x}{d t}=f(x), \quad x\left(t_{0}\right)=x_{0} ;
$$

here $t \in \mathbb{R}, x \in \mathbb{R}^{d}, f: \mathbb{R}^{d} \rightarrow \mathbb{R}^{d}$, and $d$ is the number of the system's dependent variables. The initial condition at time $t_{0}$ is denoted by $x\left(t_{0}\right)=x_{0}$.

One assumes, as usual, that $f$ has "nice" properties that guarantee the existence, uniqueness, and continuous dependence on initial states and on parameters for its solutions; in particular, we assume that $f(x)$ is continuously differentiable and that $f(x)=0$ only at isolated points, which do not form converging sequences, that is, that there are no accumulation points for the roots of $f(x)$. It is further assumed herein that there is no explicit dependence of $f$ on $t$, in which case the system is said to be autonomous. The nonautonomous case is discussed in Appendix B below.

A key concept is the notion of phase space $\mathbb{X}$, also called state space, in which the solutions of Equation A1 evolve. Here $\mathbb{X}$ is an Euclidean space of dimension $d$ whose Cartesian coordinates are given by the system's variables, $\mathbb{X}=\mathbb{R}^{d}$. For the ODE systems described in Sections 2.1 and 2.2, the dimensions are $d=3$ and 36, respectively.

The stationary solutions of Equation A1 are given by $f(x)=0$ and they are isolated points, while the evolution of the nonstationary solutions is given by a continuous succession of points, called a (phase space) trajectory. The assumptions we have made guarantee that the tangent to this phase space trajectory exists at every point and that it is simply given by $d f / d x$. The above-mentioned uniqueness theorem for ODEs ensures that-in the autonomous case at hand-trajectories cannot intersect, that is, there is a unique trajectory through any given point $x^{*}$ that is not a root of $f(x)$, that is, that satisfies $f\left(x^{*}\right) \neq 0$. This theorem implies the conservation of "the number of trajectories" or, more precisely, of their density in phase space. As a consequence, one can derive a continuity equation, called the Liouville equation, for the density of trajectories that is similar to the conservation of mass in fluid mechanics.

An autonomous, finite-dimensional dynamical system is called dissipative if the volume of any set $\mathcal{B}$ of initial states will tend to 0 as it is advected by the phase space flow in $\mathbb{X}$, with $t \rightarrow \infty$. Provided the system under investigation is dissipative, the solutions of Equation A1 will converge toward an invariant set, called an attractor, and so will the suitably defined probability densities of solution sets.

Next, one distinguishes between global and local attractors. Heuristically, a global attractor $\mathcal{A}$ is an invariant set toward which all trajectories are converging, no matter where in the phase space $\mathbb{X}$ they start, while a local attractor $\mathcal{A}^{*} \subset \mathcal{A}$ is an invariant set contained within the global attractor toward which a certain subset of trajectories that start in a subset $\mathcal{B}^{*} \subset \mathbb{X}$ are converging. Multiple local attractors may sometimes coexist. Leonov et al. (2015) provide an interesting example of multiplicity of local attractors embedded in a global attractor in the autonomous case at hand. These authors also discuss more formal definitions of attractors and demonstrate the coexistence of local attractors in the Lorenz (1963) convection model for certain parameter values.

We introduce next the Lyapunov exponents and their calculation. As in any physical system described by a set of equations of the form Equation A1, determining the initial state is prone to measurement errors. These errors can either decay or amplify depending on the solutions' stability properties. The Lyapunov exponents measure the degree of sensitivity to infinitesimal errors in the initial conditions; essentially they generalize a linear system's stability exponents to the nonlinear situation.

The existence and uniqueness of Lyapunov exponents depend on certain assumptions that form the hypothesis of the multiplicative ergodic theorem (MET: Oseledets, 1968). First proved in 1968 by V. I. Oseledets for matrix multiplication in discrete time, many generalizations of the MET to other dynamical systems-including ODE systems with both time-independent and time-dependent forcing-do exist (e.g., Ruelle, 1979, and references therein).

In a $d$-dimensional system, there are $d$ Lyapunov exponents. For the sake of simplicity and since we only need the largest one, $\sigma_{1}$, we limit ourselves to the following definition for the amplification of an infinitesimally small initial perturbation, $\delta x\left(t_{0}\right)$, 


$$
\sigma_{1}=\lim _{t-t_{0} \rightarrow \infty} \lim _{\delta x\left(t_{0}\right) \rightarrow 0} \frac{1}{t-t_{0}} \ln \left(\frac{\|\delta x(t)\|}{\left\|\delta x\left(t_{0}\right)\right\|}\right),
$$

where $\ln (\cdot)$ is the natural logarithm. If $\sigma_{1}$ is positive, then the solutions are sensitive to the errors in the initial conditions, and the system is said to display chaotic dynamics. If $\sigma_{1}=0$, then the solution is periodic, and if it is negative, the asymptotic solution is a stationary solution.

One can also define a local-in-time Lyapunov exponent, along segments of a trajectory of length $\tau$. Such a local Lyapunov exponent characterizes the local amplification of small perturbations near any point $t_{i}$ of the trajectory, and it is defined by

$$
\sigma_{1}\left(t_{i}, \tau\right)=\lim _{\delta x\left(t_{i}-\tau\right) \rightarrow 0} \frac{1}{\tau} \ln \left(\frac{\left\|\delta x\left(t_{i}\right)\right\|}{\left\|\delta x\left(t_{i}-\tau\right)\right\|}\right) .
$$

The local exponent given by Equation A 3 converges, under suitable assumptions, to Equation A2 by averaging $\sigma_{1}\left(\mathrm{t}_{\mathrm{i}}, \tau\right)$ along a very long trajectory; see, for instance, Vannitsem (2017).

\section{Appendix B: NDSs and PBAs}

The brief presentation here follows Caraballo and Han (2017). For the nonautonomous case, the paradigmatic formulation of the system is given by the initial-value problem,

$$
\frac{d x}{d t}=g(t, x), \quad x\left(t_{0}\right)=x_{0}
$$

As in Equation $\mathrm{A} 1, t \in \mathbb{R}, x \in \mathbb{R}^{d}$, and $g: \mathbb{R} \times \mathbb{R}^{d} \rightarrow \mathbb{R}^{d}$ in Equation B1, and one still assumes that $g$ has "nice" properties that guarantee the existence, uniqueness and continuous dependence on initial states and on parameters for the solutions of Equation B1. Furthermore, Caraballo and Han (2017) show that, provided the vector field $g(t, x)$ is dissipative, solutions of Equation B1 exist and satisfy the two other properties globally, that is, for all $t \in \mathbb{R}$. We call such a global solution $\varphi\left(t, t_{0}, x_{0}\right)$.

There are two key distinctions between the autonomous case and the nonautonomous one:

(1) In the autonomous setting, solutions cannot intersect, since there is only one trajectory through a given point $x_{0} \in \mathbb{R}^{d}$, due to uniqueness. Hence, for $d=2$, the only possible (forward) attracting sets are fixed points and limit cycles, that is, chaotic behavior and strange attractors can only occur for $d \geq 3$. The NDS setting is different in these respects, that is, intersections are possible at two times $t_{1}$ and $t_{2} \neq t_{1}$, and thus chaos can occur for $d=2$ and periodic forcing, as is the case, for instance, in the Van der Pol oscillator (e.g., Guckenheimer \& Holmes, 1983)

(2) In the autonomous setting, solutions depend only on the time $t-t_{0}$ elapsed since initial time, while in the NDS setting, they depend separately on the initial time $t_{0}$ and the current time $t$, at which we observe the system. In the former setting, it suffices to consider forward-in-time attraction, which results in attractors that are fixed, time-independent objects, such as fixed points, limit cycles, tori, and strange attractors, In the latter case, we need to define pullback attraction and the PBAs that it leads to

Given the uniqueness and the continuous dependence of the global solutions to Equation B1 on initial states and on parameters, it is straightforward to verify that a global solution $\varphi$ of Equation B1 satisfies:

(i) the initial value property at $t=t_{0}$, namely $\varphi\left(t_{0}, t_{0}, x_{0}\right)=x_{0}$; and

(ii) the two-parameter semigroup evolution property

$$
\varphi\left(t_{2}, t_{0}, x_{0}\right)=\varphi\left(t_{2}, t_{1}, \varphi\left(t_{1}, t_{0}, x_{0}\right)\right) \text { for } t_{0} \leq t \leq t_{2},
$$

which corresponds to the concatenation of solutions; that is, to go from $t_{0}$ to $t_{2}$ one can go first from $t_{0}$ to $t_{1}$ and then from $t_{1}$ to $t_{2}$.

One can then provide the following definition of a process. 
Definition B.1. Let $\mathbb{R}_{\geq}^{2}=\left\{\left(t, t_{0}\right) \in \mathbb{R}^{2}: t \geq t_{0}\right\}$. A process on $\mathbb{R}^{d}$ is a family of mappings

$$
\left.\varphi\left(t, t_{0}, \cdot\right)\right): \mathbb{R}^{d} \rightarrow \mathbb{R}^{d}, \quad\left(t, t_{0}\right) \in \mathbb{R}_{\geq}^{2}
$$

which satisfy

(i) the initial value property $\varphi\left(t_{0}, t_{0}, x\right)=x$ for all $x \in \mathbb{R}^{d}$ and any $t_{0} \in \mathbb{R}$

(ii) the two-parameter semigroup property for all $x \in \mathbb{R}^{d}$ and both $\left(t_{2}, t_{1}\right) \in \mathbb{R}_{\geq}^{2}$ and $\left(t_{1}, t_{0}\right) \in \mathbb{R}_{\geq}^{2}$; and

(iii) the continuity property that the mapping $\left(t, t_{0}, x\right) \mapsto \varphi\left(t, t_{0}, x\right)$ be continuous on $\mathbb{R}_{\geq}^{2} \times \mathbb{R}^{d}$

This is the so-called process formulation of an NDS. An alternative formulation is the so-called skew-product formulation, which goes back to the work of Sell, as reviewed in Sell (1971). A process as defined above is also called a two-parameter semigroup on $\mathbb{R}^{d}$, in contrast with the one-parameter semigroup of an autonomous dynamical system, since the former depends not just on the initial time $t_{0}$, as in the latter case, but also on the current time $t$.

This difference matters, in particular, in determining the asymptotic behavior of the solutions. In the autonomous case, a global solution is invariant with respect to translation, $\varphi\left(t, t_{0}, x_{0}\right)=\varphi\left(t-t_{0}, 0, x_{0}\right)$. Hence, the usual forward asymptotic behavior for $t \rightarrow+\infty$ and $t_{0}$ fixed is the same as the behavior for $t$ fixed and $t_{0} \rightarrow$ $-\infty$. This equivalence may no longer hold when the translation invariance is lost, in the NDS case.

A simple case in which analytical computations can be carried out explicitly is given in Caraballo and Han (2017, Section 3.2.1), namely

$$
\frac{d x}{d t}=-a x+b \sin t, \quad x\left(t_{0}\right)=x_{0}, \quad t \geq t_{0}
$$

Individual solutions do not have a forward limit as $t \rightarrow+\infty$ for $t_{0}$ fixed, but the difference between any two solutions vanishes in this limit. The particular solution

$$
A(t)=\frac{b(a \sin t-\cos t)}{a^{2}+1}
$$

provides the long-term information on the behavior of all the solutions of Equation B2. This result is best captured by recognizing that the pullback limit

$$
\lim _{t_{0} \rightarrow-\infty} \varphi\left(t, t_{0}, x_{0}\right)=A(t) \quad \text { for all } t \text { and } x_{0} \in \mathbb{R}
$$

yields $A(t)$ as the PBA of all the solutions of Equation B2.

One is thus led to the following rigorous definition of a PBA for a forced dissipative dynamical system subject to a time-dependent forcing, where we have generalized $\mathbb{R}^{d}$ to a finite-dimensional metric space $\mathcal{X}$ and replaced $t_{0}$ by s, for greater symmetry.

Definition B.2. A PBA is a collection $\bigcup_{t \in \mathfrak{R}} \mathcal{A}(t)$ of invariant sets that depend on time and satisfy the following conditions:

(1) For all $t, \mathcal{A}(t)$ is a compact subset in $\mathcal{X}$ that is invariant with respect to the two-parameter semi-group $\mathcal{F}(t, s)$

(2) for all $t$, pullback attraction is reached when

$$
\begin{gathered}
\mathcal{F}(t, s) \mathcal{A}(s)=\mathcal{A}(t) \quad \text { for every } s \leq t ; \quad \text { and } \\
\lim _{s \rightarrow-\infty} D_{\mathrm{H}}(\mathcal{F}(t, s) \mathcal{B}, \mathcal{A}(t))=0 \text { for all } \mathcal{B} \in \mathcal{C}
\end{gathered}
$$


where $D_{H}(E, D)$ is the Hausdorff semi-distance between two sets, and $\mathcal{C}$ is a collection of bounded sets in $\mathcal{X}$.

More general definitions for PBAs exist in infinite-dimensional spaces, like those required by the solutions of partial differential or delay-differential equations, but mathematical rigor in these cases requires more technical details (e.g., Carvalho et al., 2012; Kloeden \& Rasmussen, 2011).

The finite-dimensional definition above follows Charó et al. (2021, Appendix A and references therein). In fact, both deterministic and stochastic versions of forcing have been applied, for instance, by Checkroun et al. (2018) in the study of an infinite-dimensional, delay-differential equation model of ENSO. The deterministic forcing corresponded to the purely periodic, seasonal changes in insolation, while the stochastic component represented the westerly wind bursts appearing in other models by Timmermann and Jin (2002); see also Checkroun et al. (2011, Section 4.3).

An issue of particular interest for the properties of a PBA or of its RDS counterpart, called a random attractor (RA), is the precise nature of the interaction between the forcing and the system's intrinsic variability. This interaction is relatively simple when-in the absence of time-dependent forcing, whether deterministic or stochastic - the system has only one or more fixed points. It becomes considerably more complex when limit cycles, tori, and chaotic attractors are present. While some form of synchronization with purely periodic forcing can be expected-as in the simpler, fixed-point case-things become more complicated when the forcing or the system's intrinsic variability is more complex.

Zaslavsky (1978) already showed that periodic forcing applied to a simple two-ODE model with a limit cycle can lead to a strange attractor; see also the treatment of this case by Guckenheimer and Holmes (1983) for the Van der Pol oscillator that we mentioned at the beginning of this appendix. De Saedeleer et al. (2013) considered a Van der Pol-type oscillator subject to quasi-periodic forcing that represents an idealized version of Quaternary glaciation cycles and studied it in the framework of generalized synchronization. The results shown here in Figure 11 are somewhat reminiscent of the latter authors' results, with multistable synchronization by subsets of solutions. To the extent that the forcing in our case is chaotic, it generalizes the results of De Saedeleer et al. (2013), as well as those of Pierini and Ghil (2021) for a wind-driven ocean circulation problem with deterministically irregular but non-chaotic forcing.

In the present study, we used the forced VDDG model's Lyapunov exponents as a key tool in the systematic investigation of its PBAs obtained herein. In the PBA framework, the Lyapunov exponents of a system subject to time-dependent forcing exist and are well defined (Ruelle, 1984), provided this forcing is ergodic and unique. Since our forcing histories originate from a nonlinear oscillator with an ergodic attractor, namely the ENSO module of Section 2.1, the ergodicity of the time-dependent forcing is ensured.

As to the uniqueness, we are assuming that there are no initial errors in the tropical module's trajectory, which is independent of the extratropical module. One can also think of the Lyapunov exponent calculation as being in a $36+3$-dimensional space, with the 3-dimensional ENSO component of the trajectory being exact and not subject to divergence or convergence. We can use, therefore, the expressions (A2) and (A3) for computing the stability properties of the ENSO-forced VDDG model only. For the local exponents, we will use a value of $\tau=1$ numerical time step.

\section{Appendix C: Spectral Analyses and PBA Convergence}

\section{C1. Spectra of the Two Local PBAs}

The spectra of the solutions displayed in Figures 3 and 9 are provided in Figures $\mathrm{C} 1$ and C2, respectively. These spectra were obtained, according to the recommendations of Ghil et al. (2002), by first prefiltering the 

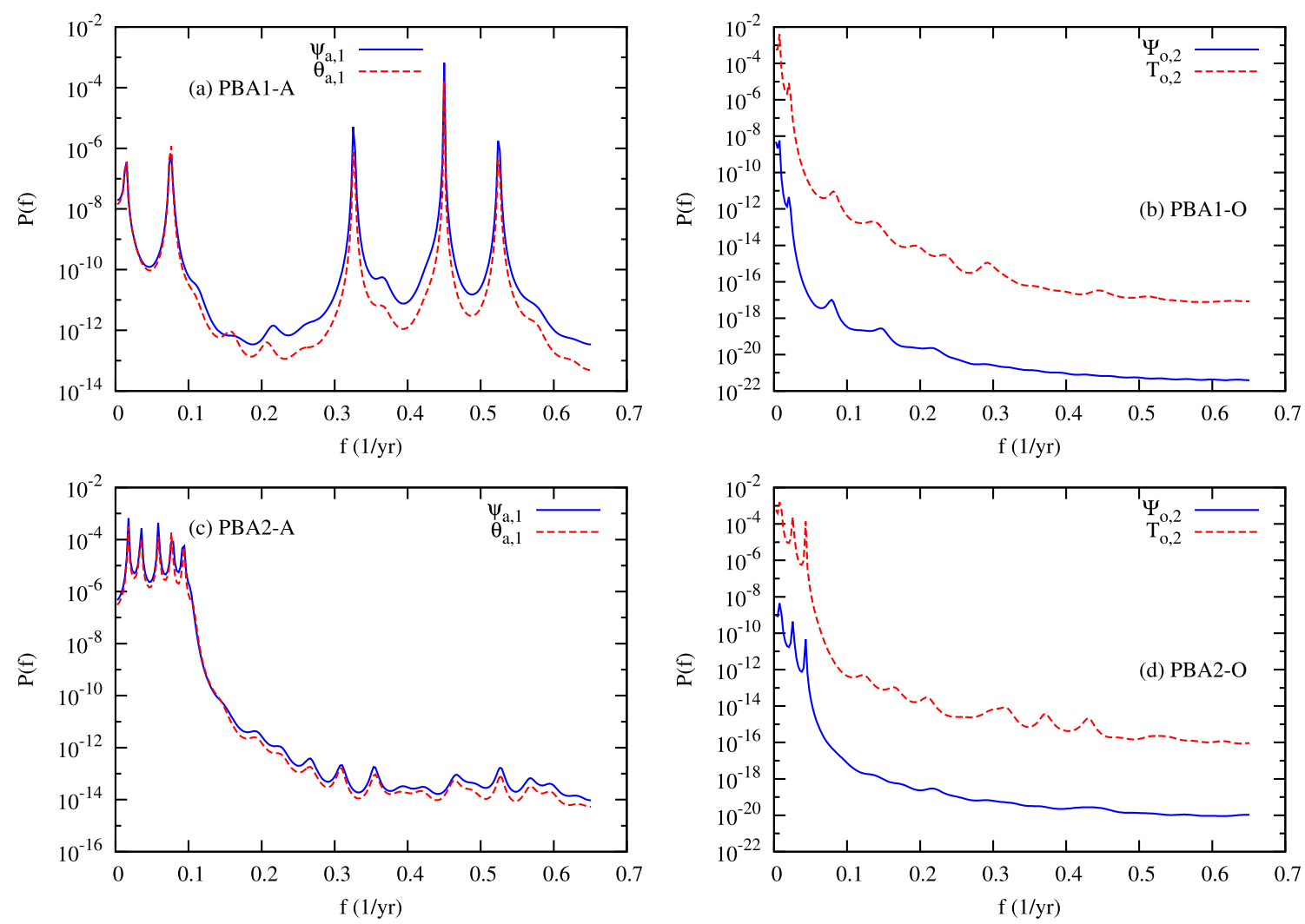

Figure C1. Spectral peaks of PBA1 and PBA2 in the periodic-forcing case. The time series analyzed correspond to the last 3,840 years of Figure 3. PBA, pullback attractor.

pairs of variables associated with the atmosphere and the oceans, namely $\left(\psi_{a, 1}, \theta_{a, 1}\right)$ and $\left(\psi_{o, 2}, T_{o, 2}\right)$, respectively, as used in Table 4. Given the results in the latter table, we retained the 10 leading RCs for subsequent analysis of the prefiltered time series by the maximum entropy method (MEM). The time series analyzed for the two figures below correspond to the last 3,840 years of Figures 3 and 9.

Penland et al. (1991) showed that this prefiltering is considerably more effective in reducing the number of spurious peaks given by MEM than the classical Akaike information criterion. The MEM order used was 40 , and the number of frequencies estimated is 256 . The calculations were carried out using the SSA-MTM Toolkit, available as freeware at https://dept.atmos.ucla.edu/tcd/ssa-mtm-toolkit.

Note that the $y$-axis in Figures $\mathrm{C} 1$ and $\mathrm{C} 2$ is logarithmic. Moreover, the peaks are fairly sharp and stand out by several orders of magnitude in most of the spectra, and by at least one order of magnitude in the least-resolved cases. The most important peaks are summarized in Table $\mathrm{C} 1$.

In the periodic-forcing case of Figure $\mathrm{C} 1$, the pair of atmospheric variables $\left(\psi_{a, 1}, \theta_{a, 1}\right)$ of PBA1 display a noticeable peak at the forcing period of roughly $P_{E} \simeq 13$ years; compare Figures 3a and C1a. The lowest-frequency peak is at a period of 66 years in this case. The forcing response at $P_{E} \simeq 13$ years is also present in the oceanic spectra of Figure C1b.

For PBA2, the picture is very different, with the atmospheric dominant peak in Figure C1c occurring at the VDDG model's intrinsic periodicity of $P_{V} \simeq 56$ years, while a peak is also present at the first harmonic thereof, with the period $P_{V} / 2 \simeq 28$ years. These peaks are not present, though, in the oceanic spectra of Figure C1d, which exhibit a very low-frequency peak at a period of about 130 years. 

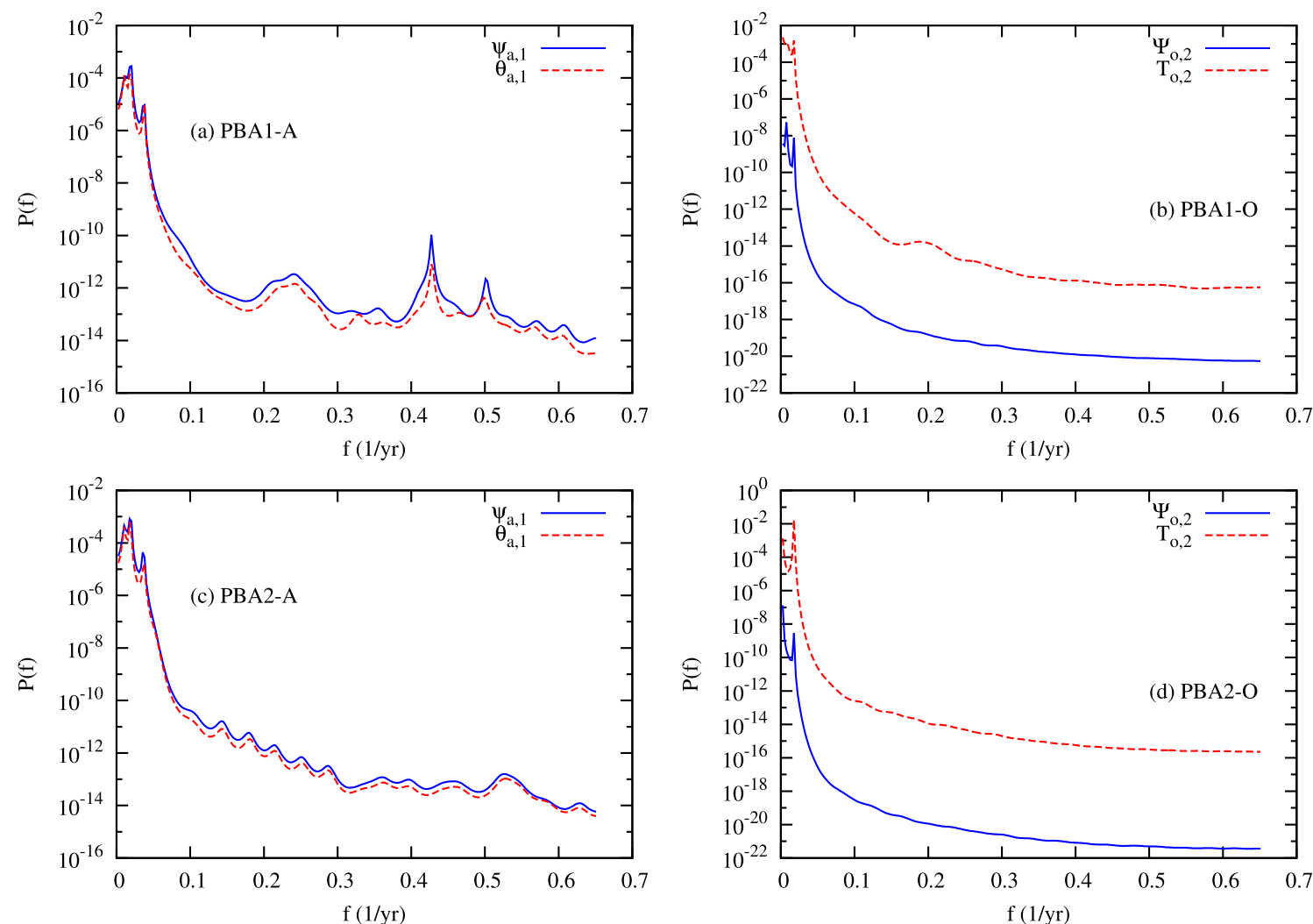

Figure C2. Spectral peaks of PBA1 and PBA2 in the chaotic-forcing case. The time series analyzed correspond to the last 3,840 years of Figure 9. PBA, pullback attractor.
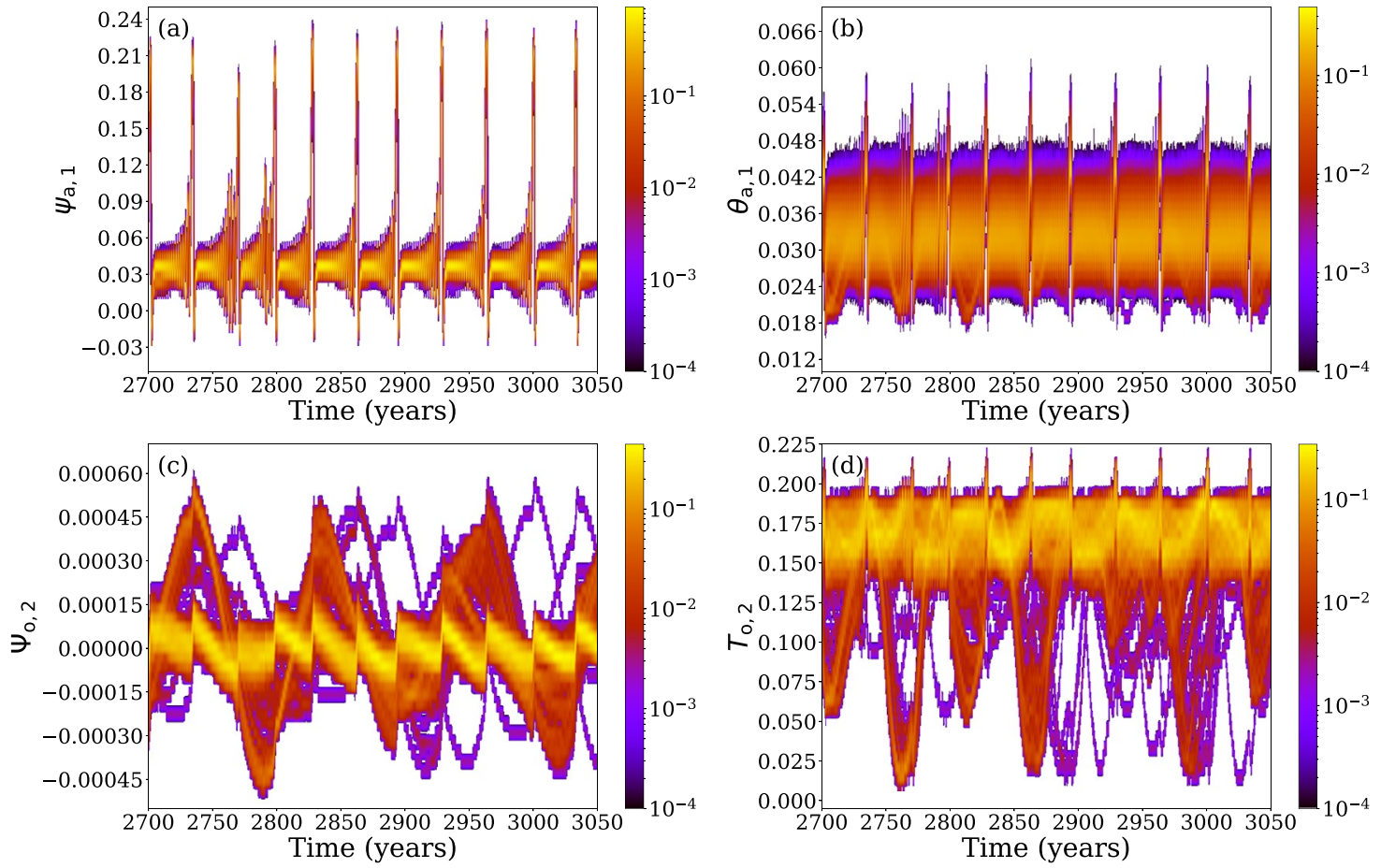

Figure C3. Solution histograms as in Figure 11, for PBA1 and 500 random initial states starting from 1,536 years in the past, instead of 3,072 years. The four panels are for the same variables: (a) $\psi_{a, 1}$; (b) $\theta_{a, 1}$; (c) $\psi_{o, 2}$; and (d) $T_{o, 2}$. PBA, pullback attractor. 


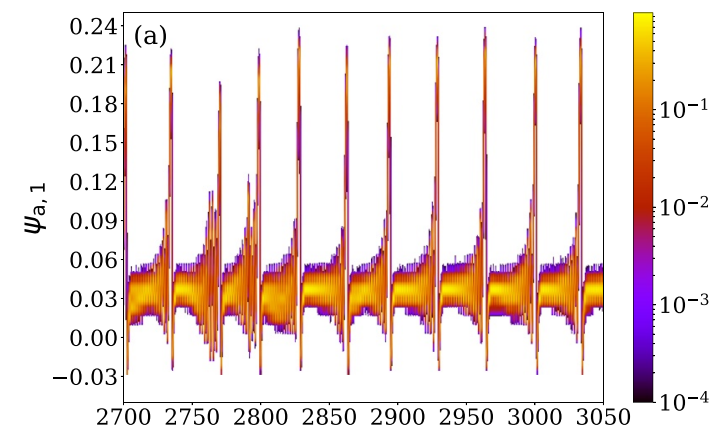
Time (years)

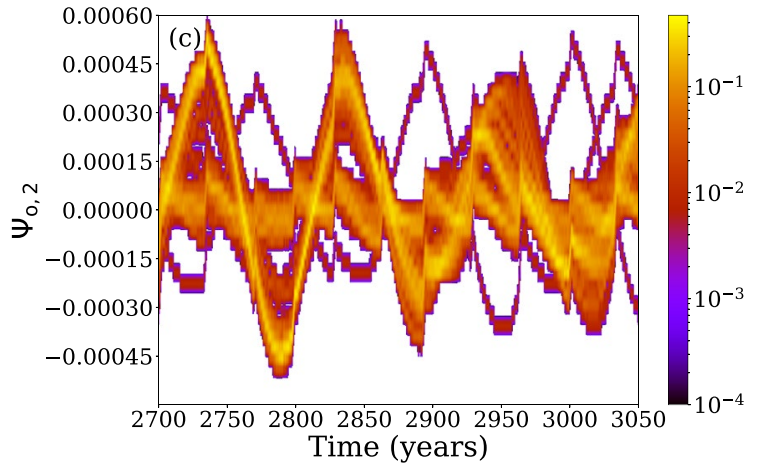

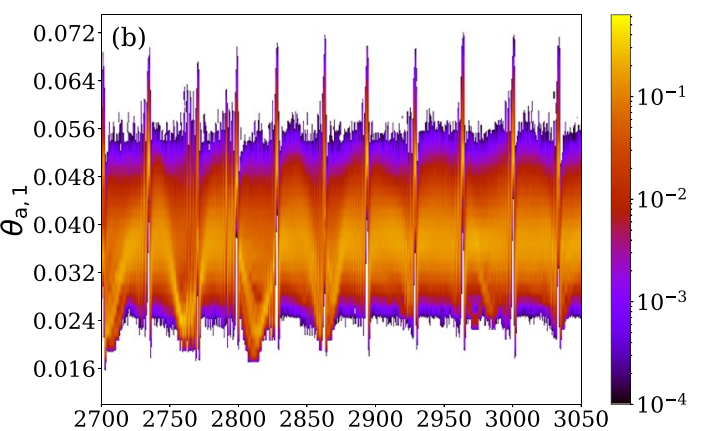
Time (years)

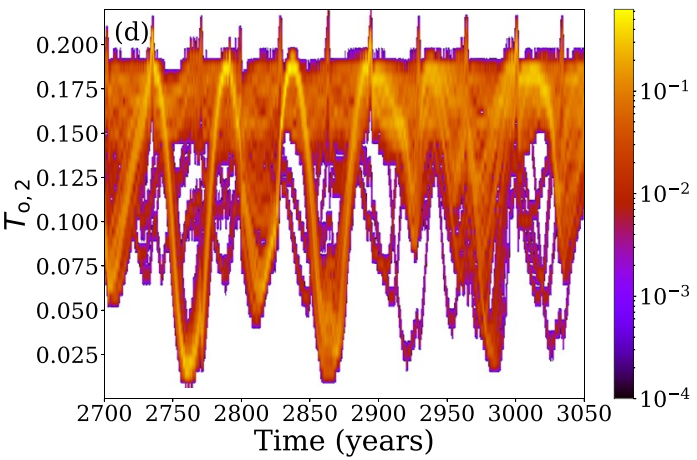

Figure C4. Same as Fig. C3, but for PBA2.

In the chaotic-forcing case of Figures $\mathrm{C} 2 \mathrm{a}$ and $\mathrm{C} 2 \mathrm{~b}$ ), there are substantial peaks at both 55-year and 28year periodicities, for PBA1 as well as PBA2, in the atmospheric variables. In this case, there's a peak at $P_{V} \simeq 56$ years in both PBA1's and PBA2's ocean, with the 130-year peak also present but in PBA2 only. The explanation for the great similarity of spectral results between the two PBAs in this case is the number of transitions between their respective basins of attractions of the two PBAs.

\section{C2. PBA Convergence}

The convergence toward the two local PBAs, PBA1, and PBA2, for the chaotic-forcing experiment is shown here with an ensemble of integrations starting from 500 random states situated at 1,536 years in the past, instead of 3,072 years. These figures should be compared to Figures 11 and 12, respectively.

Table C1

Spectral Peaks Associated With the Two Local PBAs: PBA1 and PBA2

\begin{tabular}{lccc}
\hline Forcing & Variables & PBA1 periods (yr) & PBA2 periods (yr) \\
\hline Periodic & & & \\
{$[$ Atmos $]$} & $\left(\psi_{a, 1}, \theta_{a, 1}\right)$ & $66,13,3.1,2.2,1.9$ & $56,28,17,13,11$ \\
{$[$ Ocean $]$} & $\left(\psi_{o, 2}, T_{o, 2}\right)$ & $131,49,13$ & $131,39,23$ \\
Chaotic & & & \\
{$[$ Atmos $]$} & $\left(\psi_{a, 1}, \theta_{a, 1}\right)$ & 55,28 & 55,28 \\
{$[$ Ocean $]$} & $\left(\psi_{o, 2}, T_{o, 2}\right)$ & 56 & 130,56 \\
\hline
\end{tabular}

The Length of the Time Series is $N=10,000$ Data Points and the Sampling Interval is $\Delta t=280.39$ Days, for a Total Length of 3,840 years. See Figures $\mathrm{C} 1$ and $\mathrm{C} 2$ for Details. 


\section{Data Availability Statement}

The VDDG model, as implemented in this study, can be obtained by using the Python qgs framework (Demaeyer et al., 2020). The corresponding Python code is available on Zenodo (Demaeyer \& De Cruz, 2021), as well as at https:/github.com/Climdyn/qgs. The notebooks containing the VDDG model forced by the ENSO model and the computation of the PBAs are available on Zenodo, too (Demaeyer, 2021), as well as at https://github.com/jodemaey/VDDG/ENSO/notebooks.

\section{Acknowledgments}

It is a pleasure to thank many colleagues for useful discussions on any or all of the topics brought together in this study. Associate Editor Terence O'Kane and two anonymous reviewers have substantially helped improve the presentation of the material. The study of S. Vannitsem is partially supported by the Belgian Federal Science Policy Office under contract B2/20E/P1/ ROADMAP, financed in the context of the European JPI-Climate/JPIOceans initiative. The present paper is $\mathrm{Ti}_{E} \mathrm{~S}$ contribution \# 74; this project has received funding from the EU's Horizon 2020 Research and Innovation Programme under Grant Agreement no. 820970, and it helps support the work of M. Ghil. Work on this study has also been supported by the EIT Climate-KIC (Grant no. 190733); EIT Climate-KIC is supported by the European Institute of Innovation \& Technology (EIT), a body of the European Union.

\section{References}

Alessio, S. M. (2015). Digital signal processing and spectral analysis for scientists: Concepts and applications. Springer Science \& Business Media.

Alexander, M. A., Bladé, I., Newman, M., Lanzante, J. R., Lau, N.-C., \& Scott, J. D. (2002). The atmospheric bridge: The influence of ENSO teleconnections on air-sea interaction over the global oceans. Journal of Climate, 15, 2205-2231. https://doi.org/10.1175/1520-0442(20 02) $015<2205$ :tabtio $>2.0$. co; 2

An, S.-I., \& Jin, F.-F. (2004). Nonlinearity and asymmetry of ENSO. Journal of Climate, 17, 2399-2412. https://doi. org/10.1175/1520-0442(2004)017<2399:naaoe >2.0.co;2

Arnold, L. (1998). Random dynamical systems. Springer-Verlag. https://doi.org/10.1007/978-3-662-12878-7

Arnol'd, V. I. (2012). Geometrical methods in the theory of ordinary differential equations. Springer Science \& Business Media; First Russian edition.

Ashwin, P., Wieczorek, S., Vitolo, R., \& Cox, P. (2012). Tipping points in open systems: Bifurcation, noise-induced and rate-dependent examples in the climate system. Philosophical Transactions of the Royal Society A: Mathematical, Physical and Engineering Sciences, 370(1962), 1166-1184. https://doi.org/10.1098/rsta.2011.0306

Caraballo, T., \& Han, X. (2017). Applied nonautonomous and random dynamical systems: Applied dynamical systems. Springer Science + Business Media.

Carrassi, A., Grudzien, C., Bocquet, M., Demaeyer, J., Raanes, P., \& Vannitsem, S. (2021). Data assimilation for chaotic systems. In S.-K. Park, \& X. Liang (Eds.), Data assimilation for atmospheric, oceanic and hydrological applications. Springer International Publishing, Switzerland, in press.

Carvalho, A., Langa, J. A., \& Robinson, J. (2012). Attractors for infinite-dimensional non-autonomous dynamical systems. Springer Science \& Business Media.

Charó, G. D., Chekroun, M. D., Sciamarella, D., \& Ghil, M. (2021). Topological effects of noise on nonlinear dynamics. eprint arXiv:2010.09611v5 [nlin.CD].

Checkroun, M. D., Ghil, M., \& Neelin, J. D. (2018). Pullback attractor crisis in a delay differential ENSO model. In A. A. Tsonis (Ed.), Advances in nonlinear geosciences (pp. 1-33). Springer. https://doi.org/10.1007/978-3-319-58895-7_1

Checkroun, M. D., Simmonet, E., \& Ghil, M. (2011). Stochastic climate dynamics: Random attractors and time-dependent invariant measures. Physica D: Nonlinear Phenomena, 240, 1685-1700.

De Cruz, L., Demaeyer, J., \& Vannitsem, S. (2016). The modular arbitrary-order ocean-atmosphere model: MAOOAM v1.0. Geoscientific Model Development, 9(8), 2793-2808. https://doi.org/10.5194/gmd-9-2793-2016

De Cruz, L., Schubert, S., Demaeyer, J., Lucarini, V., \& Vannitsem, S. (2018). Exploring the Lyapunov instability properties of high-dimensional atmospheric and climate models. Nonlinear Processes in Geophysics, 25(2), 387-412. https://doi.org/10.5194/npg-25-387-2018

Demaeyer, J. (2021). Vddg enso notebooks: Version 1.1 release. Zenodo. Retrieved from https://doi.org/10.5281/zenodo.4728195

Demaeyer, J., \& De Cruz, L. (2021). qgs: Version 0.2.2 release. Zenodo. Retrieved from https://doi.org/10.5281/zenodo.4587665,

Demaeyer, J., De Cruz, L., \& Vannitsem, S. (2020). qgs: A flexible Python framework of reduced-order multiscale climate models. Journal of Open Source Software, 5(56), 2597. https://doi.org/10.21105/joss.02597

Demaeyer, J., \& Vannitsem, S. (2018). Comparison of stochastic parameterizations in the framework of a coupled ocean-atmosphere model. Nonlinear Processes in Geophysics, 25(3), 605-631. https://doi.org/10.5194/npg-25-605-2018

De Saedeleer, B., Crucifix, M., \& Wieczorek, S. (2013). Is the astronomical forcing a reliable and unique pacemaker for climate? A conceptual model study. Climate Dynamics, 40, 273-294. https://doi.org/10.1007/s00382-012-1316-1

Dijkstra, H. A., \& Ghil, M. (2005). Low-frequency variability of the large-scale ocean circulation: A dynamical systems approach. Reviews of Geophysics, 43(3), RG3002. https://doi.org/10.1029/2002RG000122

Ditlevsen, P. D., \& Ashwin, P. (2018). Complex climate response to astronomical forcing: The Middle-Pleistocene Transition in glacial cycles and changes in frequency locking. Frontiers in Physics, 6. https://doi.org/10.3389/fphy.2018.00062

Drótos, G., Bódai, T., \& Tél, T. (2015). Probabilistic concepts in a changing climate: A snapshot attractor picture. Journal of Climate, 28, 3275-3288. https://doi.org/10.1175/jcli-d-14-00459.1

Drótos, G., Bódai, T., \& Tél, T. (2016). Quantifying nonergodicity in nonautonomous dissipative dynamical systems: An application to climate change. Physical Review E - Statistical Physics, Plasmas, Fluids, and Related Interdisciplinary Topics, 94, 022214. https://doi. org/10.1103/PhysRevE.94.022214

Ghil, M. (1994). Cryothermodynamics: The chaotic dynamics of paleoclimate. Physica D, 77, 130-159. https://doi. org/10.1016/0167-2789(94)90131-7

Ghil, M. (2001). Hilbert problems for the geosciences in the 21st century. Nonlinear Processes in Geophysics, 8(4/5), 211. https://doi. org/10.5194/npg-8-211-2001

Ghil, M. (2019). A century of nonlinearity in the geosciences. Earth and Space Science, 6, 1007-1042. https://doi.org/10.1029/2019EA000599

Ghil, M., Allen, M. R., Dettinger, M. D., Ide, K., Kondrashov, D., Mann, M. E., \& Yiou, P. (2002). Advanced spectral methods for climatic time series. Reviews of Geophysics, 40(1), 41. https://doi.org/10.1029/2000RG000092

Ghil, M., Chekroun, M. D., \& Simonnet, E. (2008). Climate dynamics and fluid mechanics: Natural variability and related uncertainties. Physica D: Nonlinear Phenomena, 237(14-17), 2111-2126. https://doi.org/10.1016/j.physd.2008.03.036

Ghil, M., \& Childress, S. (1987). Topics in geophysical fluid dynamics: Atmospheric dynamics, dynamo theory, and climate dynamics. Springer Science+Business Media. 
Ghil, M., \& Lucarini, V. (2020). The physics of climate variability and climate change. Reviews of Modern Physics, 92(3), 035002. https:// doi.org/10.1103/revmodphys.92.035002

Ghil, M., \& Vautard, R. (1991). Interdecadal oscillations and the warming trend in global temperature time series. Nature, 350, 324-327. https://doi.org/10.1038/350324a0

Gill, A. E. (1982). Atmosphere-ocean dynamics. Academic Press.

Groth, A., Feliks, Y., Kondrashov, D., \& Ghil, M. (2017). Interannual variability in the North Atlantic Ocean's temperature field and its association with the wind stress forcing. Journal of Climate, 30(7), 2655-2678. https://doi.org/10.1175/jcli-d-16-0370.1

Guckenheimer, J., \& Holmes, P. J. (1983). Nonlinear oscillations, dynamical systems, and bifurcations of vector fields. Springer Science \& Business Media. https://doi.org/10.1007/978-1-4612-1140-2

Guckenheimer, J., Timmermann, A., Dijkstra, H., \& Roberts, A. (2017). (Un)predictability of strong El Niño events. Dynamics and Statistics of the Climate System, 2. https://doi.org/10.1093/climsys/dzx004

Held, I. M. (2005). The gap between simulation and understanding in climate modeling. Bulletin of the American Meteorological Society, 86(11), 1609-1614. https://doi.org/10.1175/bams-86-11-1609

Hoerling, M. P., \& Kumar, A. (2002). Atmospheric response patterns associated with tropical forcing. Journal of Climate, 15, $2184-2203$. https://doi.org/10.1175/1520-0442(2002)015<2184:ARPAWT>2.0.CO;2

IPCC. (2014). Climate change 2013: The physical science basis, In T. Stocker (Ed.), In Contribution of working group I to the fifth assessment report of the intergovernmental panel on climate change. Cambridge University Press. https://doi.org/10.1017/cbo9781107415324

Jin, F.-F. (1996). Tropical ocean-atmosphere interaction, the Pacific cold tongue, and the El-Niño-Southern Oscillation. Science, 274, 76-78. https://doi.org/10.1126/science.274.5284.76

Jin, F.-F. (1997). An equatorial ocean recharge paradigm for ENSO. Part I: Conceptual model. Journal of the Atmospheric Sciences, 54, 811-829. https://doi.org/10.1175/1520-0469(1997)054<0811:aeorpf >2.0.co;2

Jin, F.-F., Neelin, J. D., \& Ghil, M. (1994). El Niño on the devil's staircase: Annual subharmonic steps to chaos. Science, 264, 70-72. https:// doi.org/10.1126/science.264.5155.70

Jin, F.-F., Neelin, J. D., \& Ghil, M. (1996). El Niño/Southern Oscillation and the annual cycle: Subharmonic frequency-locking and aperiodicity. Physica D: Nonlinear Phenomena, 98, 442-465. https://doi.org/10.1016/0167-2789(96)00111-x

Kimoto, M., \& Ghil, M. (1993). Multiple flow regimes in the Northern Hemisphere winter. Part II: Sectorial regimes and preferred transitions. Journal of the Atmospheric Sciences, 50, 2645-2673. https://doi.org/10.1175/1520-0469(1993)050<2645:mfritn>2.0.co;2

Kloeden, P. E., \& Rasmussen, M. (2011). Nonautonomous dynamical systems. American Mathematical Society. https://doi.org/10.1090/ surv/176

Kravtsov, S., Grimm, C., \& Gu, S. (2018). Global-scale multidecadal variability missing in state-of-the-art climate models. Climate and Atmospheric Science, 1(1), 34. https://doi.org/10.1038/s41612-018-0044-6

Kumar, A., \& Hoerling, M. P. (1995). Prospects and limitations of seasonal atmospheric GCM predictions. Bulletin of the American Meteorological Society, 76, 335-345. https://doi.org/10.1175/1520-0477(1995)076<0335:PALOSA > 2.0.CO;2

Kuptsov, P. V., \& Parlitz, U. (2012). Theory and computation of covariant Lyapunov vectors. Journal of Nonlinear Science, 22(5), 727-762. https://doi.org/10.1007/s00332-012-9126-5

Legras, B., \& Ghil, M. (1985). Persistent anomalies, blocking, and variations in atmospheric predictability. Journal of the Atmospheric Sciences, 42, 433-471. https://doi.org/10.1175/1520-0469(1985)042<0433:pabavi $>2.0 . c 0 ; 2$

Leonov, G.A., Kuznetsov, N.V.,\& Mokaev,T.N.(2015).Homoclinicorbits, and self-excited and hidden attractorsin a Lorenz-likesystem describing convective fluid motion. The European Physical Journal - Special Topics, 224, 1421-1458. https://doi.org/10.1140/epjst/e2015-02470-3

Le Treut, H., Portes, J., Jouzel, J., \& Ghil, M. (1988). Isotopic modeling of climatic oscillations: Implications for a comparative study of marine and ice-core records. Journal of Geophysical Research, 93, 9365-9383. https://doi.org/10.1029/jd093id08p09365

López-Parages, J., Rodríguez-Fonseca, B., Dommenget, D., \& Frauen, C. (2016). ENSO influence on the North Atlantic European climate: A non-linear and non-stationary approach. Climate Dynamics, 47, 2071-2084. https://doi.org/10.1007/s00382-015-2951-0

Lorenz, E. N. (1963). Deterministic nonperiodic flow. Journal of the Atmospheric Sciences, 20, 130-141. https://doi. org/10.1175/1520-0469(1963)020<0130:dnf > 2.0.co;2

Lorenz, E. N. (1990). Can chaos and intransitivity lead to interannual variability?. Tellus A, 42(3), 378-389. https://doi. org/10.1034/j.1600-0870.1990.t01-2-00005.x

Lucarini, V., \& Bódai, T. (2017). Edge states in the climate system: Exploring global instabilities and critical transitions. Nonlinearity, 30(7), R32-R66. https://doi.org/10.1088/1361-6544/aa6b11

McPhaden, M. J., Santoso, A., \& Cai, W.(Eds.), (2020). El Niño Southern Oscillation in a changing climate. John Wiley \& Sons

Nicolis, G. (1995). Introduction to nonlinear science. Cambridge University Press. https://doi.org/10.1017/cbo9781139170802

Nidheesh, A. G., Lengaigne, M., Vialard, J., Izumo, T., Unnikrishnan, A. S., \& Cassou, C. (2017). Influence of ENSO on the Pacific decadal oscillation in CMIP models. Climate Dynamics, 49, 3309-3326. https://doi.org/10.1007/s00382-016-3514-8

O'Reilly, C. H., Weisheimer, A., Woollings, T., Gray, L. J., \& MacLeod, D. (2019). The importance of stratospheric initial conditions for winter North Atlantic Oscillation predictability and implications for the signal-to-noise paradox. Quarterly Journal of the Royal Meteor ological Society, 145(718), 131-146. https://doi.org/10.1002/qj.3413

Oseledets, V. I. (1968). A multiplicative ergodic theorem: Lyapunov characteristic numbers for dynamical systems. Transactions of the Moscow Mathematical Society, 19, 197-231.

Pedlosky, J. (1987). Geophysical fluid dynamics (2nd ed.). Springer-Verlag. https://doi.org/10.1007/978-1-4612-4650-3

Penland, C., Ghil, M., \& Weickmann, K. M. (1991). Adaptive filtering and maximum entropy spectra with application to changes in atmospheric angular momentum. Journal of Geophysical Research, 96(D12), 22659-22671. https://doi.org/10.1029/91jd02107

Penny, S., Bach, E., Bhargava, K., Chang, C.-C., Da, C., Sun, L., \& Yoshida, T. (2019). Strongly coupled data assimilation in multiscale media: Experiments using a quasi-geostrophic coupled model. Journal of Advances in Modeling Earth Systems, 6, 1803-1829. https:// doi.org/10.1029/2019ms001652

Philander, S. (1990). El Niño and the Southern Oscillation. Academic Press.

Pierini, S. (2020). Statistical significance of small ensembles of simulations and detection of the internal climate variability: An excitable ocean system case study. Journal of Statistical Physics, 179, 1475-1495. https://doi.org/10.1007/s10955-019-02409-x

Pierini, S., Chekroun, M. D., \& Ghil, M. (2018). The onset of chaos in nonautonomous dissipative dynamical systems: A low-order ocean-model case. Nonlinear Processes in Geophysics, 25, 671-692. https://doi.org/10.5194/npg-25-671-2018

Pierini, S., \& Ghil, M. (2021). Climate tipping points induced by parameter drift: An excitable system study. Scientific Reports. https://doi. org/10.1038/s41598-021-90138-1 
Pierini, S., Ghil, M., \& Chekroun, M. D. (2016). Exploring the pullback attractors of a low-order quasigeostrophic ocean model: The deterministic case. Journal of Climate, 29, 4185-4202. https://doi.org/10.1175/jcli-d-15-0848.1

Roberts, A., Guckenheimer, J., Widiasih, E., Timmermann, A., \& Jones, C. K. R. T. (2016). Mixed-mode oscillations of El Niño-Southern Oscillation. Journal of the Atmospheric Sciences, 73, 1755-1766. https://doi.org/10.1175/jas-d-15-0191.1

Ruelle, D. (1979). Ergodic theory of differentiable dynamical systems. Publications Mathématiques Institut des Hautes Études Scientifiques, 50, 275-306. https://doi.org/10.1007/bf02684768

Ruelle, D. (1984). Characteristic exponents for a viscous fluid subjected to time dependent forces. Communications in Mathematical Physics, 93, 285-300. https://doi.org/10.1007/bf01258529

Schemm, S., Rivière, G., Ciasto, L. M., \& Li, C. (2018). Extratropical cyclogenesis changes in connection with tropospheric ENSO teleconnections to the North Atlantic: Role of stationary and transient waves. Journal of the Atmospheric Sciences, 75, 3943-3964. https://doi. org/10.1175/JAS-D-17-0340.1

Sell, G. R. (1971). Topological dynamics and ordinary differential equations. Van Nostrand Reinhold.

Sévellec, F., \& Fedorov, A. V. (2015). Unstable AMOC during glacial intervals and millennial variability: The role of mean sea ice extent. Earth and Planetary Science Letters, 429, 60-68. https://doi.org/10.1016/j.epsl.2015.07.022

Smith, D. M., Scaife, A. A., Eade, R., Athanasiadis, P., Bellucci, A., Bethke, I., et al. (2020). North Atlantic climate far more predictable than models imply. Nature, 583, 796-800. https://doi.org/10.1038/s41586-020-2525-0

Tél, T., Bódai, T., Drótos, G., Haszpra, T., Herein, M., Kaszás, B., \& Vincze, M. (2020). The theory of parallel climate realizations. Journal of Statistical Physics, 179, 1496-1530. https://doi.org/10.1007/s10955-019-02445-7

Timmermann, A., \& Jin, F.-F. (2002). A nonlinear mechanism for decadal El Niño amplitude changes. Geophysical Research Letters, 29(1), 3-1-3-4. https://doi.org/10.1029/2001GL013369

Timmermann, A., Jin, F.-F., \& Abshagen, J. (2003). A nonlinear theory for El Niño bursting. Journal of the Atmospheric Sciences, 60, 152-165. https://doi.org/10.1175/1520-0469(2003)060<0152:antfen>2.0.co;2

Tondeur, M., Carrassi, A., Vannitsem, S., \& Bocquet, M. (2020). On temporal scale separation in coupled data assimilation with the ensemble Kalman filter. Journal of Statistical Physics, 179, 1161-1185. https://doi.org/10.1007/s10955-020-02525-Z

Tziperman, E., Stone, L., Cane, M. A., \& Jarosh, H. (1994). El Niño chaos: Overlapping of resonances between the seasonal cycle and the Pacific ocean-atmosphere oscillator. Science, 264, 72-74. https://doi.org/10.1126/science.264.5155.72

Vannitsem, S. (2017). Predictability of large-scale atmospheric motions: Lyapunov exponents and error dynamics. Chaos, $27,32101$. https://doi.org/10.1063/1.4979042

Vannitsem, S., Demaeyer, J., De Cruz, L., \& Ghil, M. (2015). Low-frequency variability and heat transport in a low-order nonlinear coupled ocean-atmosphere model. Physica D: Nonlinear Phenomena, 309, 71-85. https://doi.org/10.1016/j.physd.2015.07.006

Vannitsem, S., \& Ghil, M. (2017). Evidence of coupling in ocean-atmosphere dynamics over the North Atlantic. Geophysical Research Letters, 44(4), 2016-2026.

Vannitsem, S., \& Lucarini, V. (2016). Statistical and dynamical properties of covariant Lyapunov vectors in a coupled atmosphere-ocean model - Multiscale effects, geometric degeneracy, and error dynamics. Journal of Physics A: Mathematical and Theoretical, 49(22), 224001. https://doi.org/10.1088/1751-8113/49/22/224001

Varadi, F., Ghil, M., \& Kaula, W. M. (1999). Jupiter, Saturn, and the edge of chaos. Icarus, 139(2), 286-294. https://doi.org/10.1006/ icar.1999.6104

Wilson, C. (1985). The great inequality of Jupiter and Saturn: From Kepler to Laplace. Archive for History of Exact Sciences, 33(1-3), 15-290. https://doi.org/10.1007/bf00328048

Young, L.-S. (2017). Generalizations of SRB measures to nonautonomous, random, and infinite dimensional systems. Journal of Statistical Physics, 166(3-4), 494-515. https://doi.org/10.1007/s10955-016-1639-0

Zaslavsky,G.M.(1978).Thesimplestcase of astrangeattractor.PhysicsLettersA,69(3),145-147.https://doi.org/10.1016/0375-9601(78)90195-0 